Research Paper

\title{
NAP1L1 promotes proliferation and chemoresistance in glioma by inducing CCND1/CDK4/CDK6 expression through its interaction with HDGF and activation of c-Jun
}

\author{
Zigui Chen ${ }^{1,2,}{ }^{*}$, Yingying Xie ${ }^{1,2}$, Hongcheng Luo ${ }^{3}$, Ye Song ${ }^{4}$, Tianshi Que ${ }^{4}$, Rentong Hu ${ }^{3}$, Huatuo \\ Huang ${ }^{3}$, Kunxiang Luo ${ }^{5}$, Chuanyu Li $^{5}$, Chengjian Qin ${ }^{5}$, Chuanhua Zheng ${ }^{5}$, Weiyi Fang ${ }^{1,2}$, Longyang \\ Liu $^{1,2}$, Hao Long ${ }^{1,4}$, Qisheng Luo ${ }^{1,5}$ \\ ${ }^{1}$ Neuroscience Center, Cancer Center, Integrated Hospital of Traditional Chinese Medicine, Southern Medical \\ University, Guangzhou 510315, China \\ ${ }^{2}$ Southern Medical University, Guangzhou, Guangdong 510000, China \\ ${ }^{3}$ Department of Laboratory Medicine, Affiliated Hospital of Youjiang Medical University for Nationalities, Guangxi, \\ Baise 53300, China \\ ${ }^{4}$ Department of Neurosurgery, Nanfang Hospital, Southern Medical University, Guangzhou, Guangdong 510515, \\ China \\ ${ }^{5}$ Department of Neurosurgery, Affiliated Hospital of Youjiang Medical University for Nationalities, Guangxi, Baise \\ 53300, China \\ ${ }^{*}$ First author
}

Correspondence to: Weiyi Fang, Longyang Liu, Hao Long, Qisheng Luo; email: Iz1980@i.smu.edu.cn, 1 ly2020@smu.edu.cn, longhao@smu.edu.cn, 1917@ymcn.edu.cn

Keywords: NAP1L1, HDGF, glioma, proliferation, chemoresistance

Received: September 7, $2021 \quad$ Accepted: December 13, 2021

Published: December 27, 2021

Copyright: (C) 2021 Chen et al. This is an open access article distributed under the terms of the Creative Commons Attribution License (CC BY 3.0), which permits unrestricted use, distribution, and reproduction in any medium, provided the original author and source are credited.

\section{ABSTRACT}

The prognosis of glioma is poor as its pathogenesis and mechanisms underlying cisplatin chemoresistance remain unclear. Nucleosome assembly protein 1 like 1 (NAP1L1) is regarded as a hallmark of malignant tumors. However, the role of NAP1L1 in glioma remains unknown. In this study, we aimed to investigate the molecular functions of NAP1L1 in glioma and its involvement in cisplatin chemoresistance, if any. NAP1L1 was found to be upregulated in samples from The Cancer Genome Atlas (TCGA) database. Immunohistochemistry indicated that NAP1L1 and hepatoma-derived growth factor (HDGF) were enhanced in glioma as compared to the para-tumor tissues. High expressions of NAP1L1 and HDGF were positively correlated with the WHO grade, KPS, Ki-67 index, and recurrence. Moreover, NAP1L1 expression was also positively correlated with the HDGF expression in glioma tissues. Functional studies suggested that knocking down NAP1L1 could significantly inhibit glioma cell proliferation both in vitro and in vivo, as well as enhance the sensitivity of glioma cells to cisplatin (cDDP) in vitro. Mechanistically, NAP1L1 could interact with HDGF at the protein level and they co-localize in the cytoplasm. HDGF knockdown in NAP1L1-overexpressing glioma cells significantly inhibited cell proliferation. Furthermore, HDGF could interact with c-Jun, an oncogenic transcription factor, which eventually induced the expressions of cell cycle promoters, CCND1/CDK4/CDK6. This finding suggested that NAP1L1 could interact with HDGF, and the latter recruited c-Jun, a key oncogenic transcription factor, that further induced CCND1/CDK4/CDK6 expression, thereby promoting proliferation and chemoresistance in glioma cells. High expression of NAP1L1 in glioma tissues indicated shorter overall survival in glioma patients. 


\section{INTRODUCTION}

Glioma is the most commonly diagnosed intracranial malignancy. Currently, extended surgical resection combined with radiotherapy or chemotherapy is the main treatment strategies [1]. Due to the heterogeneity of tumor-related genes and tumor susceptibility, the prognosis of patients with glioma remains poor, thereby making it a big problem among patients with intracranial tumors [2,3]. Despite the novel therapeutic approaches targeted for glioma treatment, the death rate remains consistently high and their 5-year prognosis remains low. The poor effects of therapy may be attributed to the high proliferation rates of glioma cells and their resistance to chemotherapy. Therefore, it is important to identify novel molecular markers and develop corresponding treatment strategies to improve the prognosis of patients with glioma.

Nucleosome Assembly Protein 1 Like 1, NAP1L1, also known as NRP or NAP1, is located at $12 \mathrm{q} 21.2$ and belongs to the family of nucleosome assembly proteins. NAP1L1 is present in most human tissues and cell lines, however, upregulated levels are often detected in rapidly proliferating cells [4]. Several studies report the high NAP1L1 expression in tumors [5-8], which points to its potential role in these malignancies. Although as early as the 1990s, Japanese scientists showed that NAP1 could accelerate the assembly of nucleosomes and participate in cell DNA replication and chromatin regulation [9], it is only in recent years that studies have shown its close association with cell proliferation and other functions in stem cells. Nevertheless, the biological role as well as the molecular mechanism of NAP1L1 underlying glioma onset and progression remains unknown. In our previous study, NAP1L1 was found to be significantly upregulated in glioma as compared to the para-tumor tissues and high NAP1L1 expression was associated with poor survival in patients with glioma. NAP1L1 is an oncogene, and its knockdown inhibits glioma cell proliferation. But the comprehensive molecular mechanisms of NAP1L1 underlying glioma onset and progression remain unclear. These results would provide new insights to illustrate the molecular mechanism of glioma development.

In this study, we investigated specific molecular functions and roles of NAP1L1 in glioma cells, thereby providing evidence that NAP1L1 could promote cell proliferation and chemoresistance in glioma cells by interacting with HDGF and subsequently, activating cJun to induce CCND1/CDK4/CDK6 expressions. Our findings implicated NAP1L1 as a potential diagnostic biomarker and therapy target for glioma.

\section{RESULTS}

NAP1L1 is strongly expressed in glioma tissues and correlates with low prognosis

Several previous studies show that NAP1L1 expression is strictly correlated with malignant neoplastic progression in several tumor types [5-8]. Based on the analysis of samples from the TCGA database, we found increased expression of NAP1L1 at the mRNA level (Figure 1A). Analysis of OS and disease free survival (DFS) showed that overexpression of NAP1L1 was a negative factor that decreased the overall survival duration in glioma patients (Figure 1B). The RT-qPCR analysis also confirmed that NAP1L1 mRNA expression was significantly enhanced in 24 gliomas tissues as compared to the 24 corresponding para-tumor tissues $(P<0.001)$ (Figure $1 C)$. A tissue microarray (TMA) with 108 glioma tissue samples and 24 para-tumor tissues was used to assess the expression level of NAP1L1 (Figure 1D, 1E). The survival analysis demonstrated that overexpression of NAP1L1 was an adverse factor that decreased the survival duration of glioma patients (Figure 1F, 1G). As shown in Table 1, high expression of NAP1L1 in glioma tissues was significantly related to WHO grades I IV, KPS $<80$, Ki-67 index $\geq 20 \%$, and recurrence. Furthermore, univariate and multivariate cox regression analyses showed that NAP1L1 was a disadvantage predictive factor, which indicated poor survival in glioma patients (Tables 2 and 3 ).

\section{Suppressing NAP1L1 inhibits glioma cell proliferation and chemoresistance}

To study the potential role of NAP1L1 in the progression of glioma, we transfected LN229 and U87 cells with lentiviral constructs expressing short hairpin RNA targeting NAP1L1 and the corresponding negative control (shNC) (Supplementary Figure 1A). The protein level expression of NAP1L1 was assessed by western blotting (Figure 2A). After confirming efficient knockdown using shNAP1L1 in the LN229 cell line, the same fragment in the U87 cell line was separately detected by RT-PCR assay and compared with the shNC group (Figure 2A). We also assessed the interference effects from small interfering RNAs (siRNAs) that were used to knock down the endogenous NAP1L1 as compared to the negative control (si-NC) group (Figure 2B).

Next, we investigated the effect of attenuated expression of NAP1L1 on the growth of glioma cells in vitro. The MTT assay demonstrated that NAP1L1 knockdown could significantly decrease the glioma cells proliferation as compared to that of the control (si-NC) group (Figure 2C). In addition, clone formation assays further confirmed that shNAP1L1-mediated 
knockdown in glioma cells significantly inhibited cell proliferation (Figure 2D). Moreover, a model of subcutaneous xenograft was established in nude mice with shNAP1L1-transfected glioma cells. We found that NAP1L1 knockdown, that is, in mice that carried the shNAP1L1 glioma cells, resulted in the formation of smaller tumors as compared to those in the control (sh-NC) group (Figure 2E-2G).

Interestingly, glioma cells with stably silenced NAP1L1 showed significantly enhanced sensitivity to cisplatin
(DDP) treatment. Glioma cells were processed with various concentrations of DDP after $48 \mathrm{~h}$ and the cell growth inhibition rates were calculated after NAP1L1 silencing. The IC50 value of DDP was $3.38 \mu \mathrm{M}$ in the parental LN229 cells but decreased significantly to $1.56 \mu \mathrm{M}$ in NAP1L1-silenced LN229 cells $(P<0.05)$; a similar IC50 reduction from $4.84 \mu \mathrm{M}$ to $2.58 \mu \mathrm{M}$ was observed in the U87 cells upon silencing (Figure 2H). Moreover, silenced NAP1L1 also increased sensitivity to temozolomide and the results were showed in Supplementary Figure 1B.
A

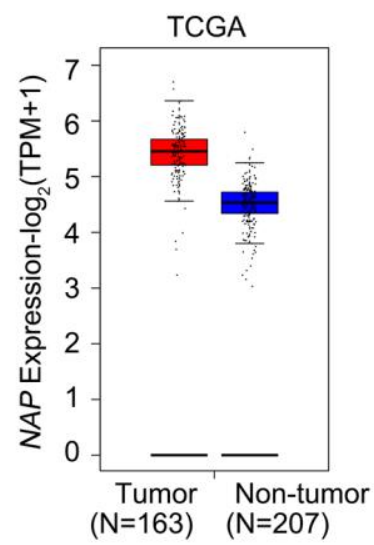

C

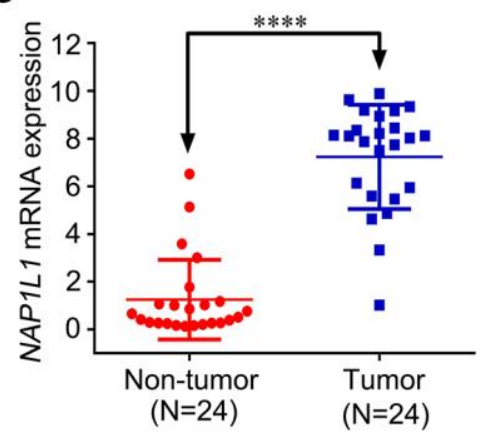

E

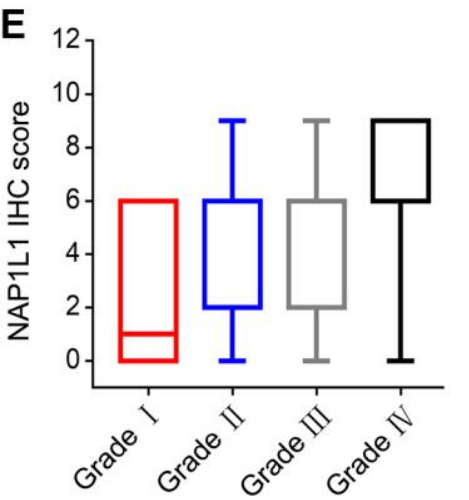

B

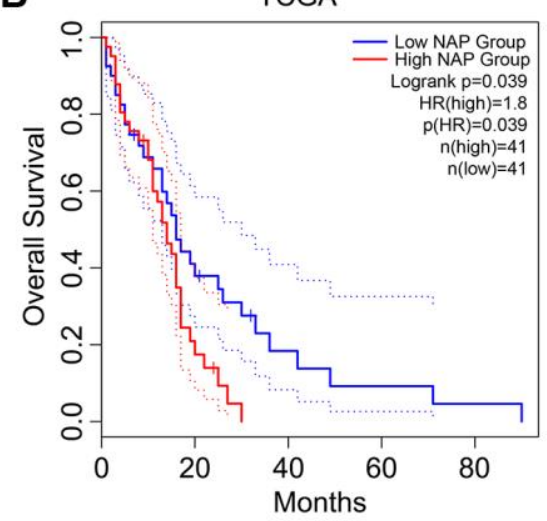

D

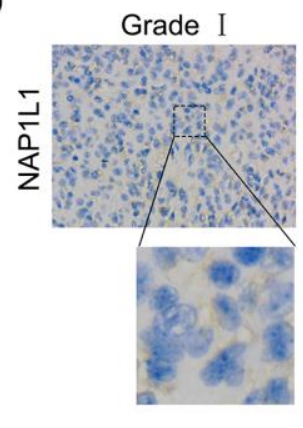

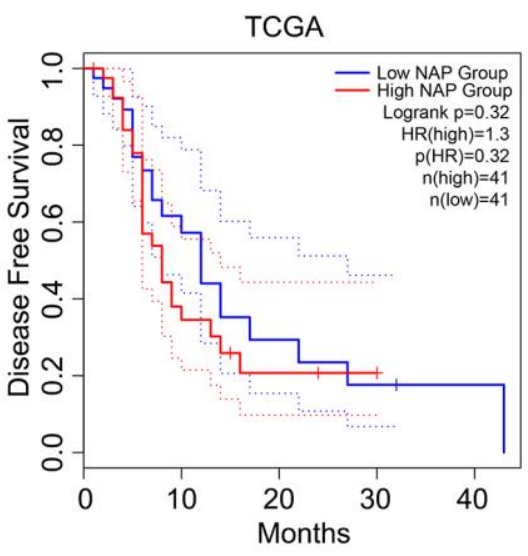

Grade II
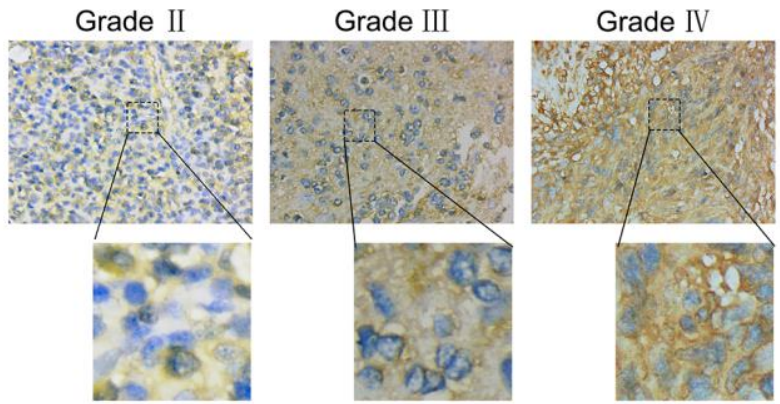

G

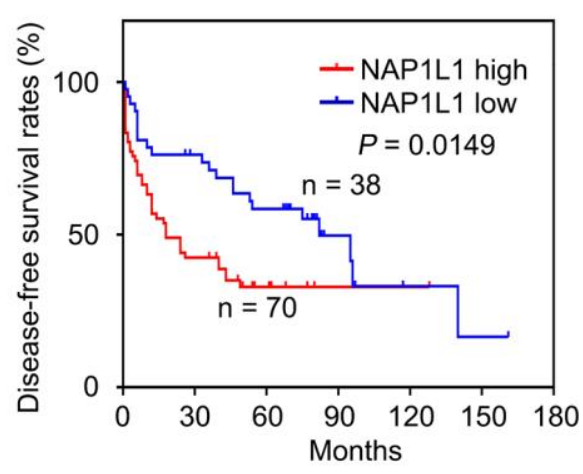

Figure 1. NAP1L1 is highly expressed in glioma and correlates with poor prognosis. (A) NAP1L1 mRNA expression in glioma tissues and para-tumor tissues among the glioma patients obtained from the TCGA database. (B) Kaplan-Meier survival analysis for overall survival based on the NAP1L1 expression data. (C) RT-qPCR analysis of NAP1L1 mRNA expression in 24 glioma tissues and 24 para-tumor tissues. (D, E) Representative images of NAP1L1 staining (D) and IHC score (E) of in (grade I-IV) glioma tissues (scale bar: $50 \mu \mathrm{m})$. (F, G) Kaplan-Meier survival analysis for overall survival (F) and Disease Free survival (G) in TMA showing NAP1L1 expression. Data are presented as the mean \pm SD for three independent experiments. ${ }^{*} P<0.05,{ }^{* *} P<0.01,{ }^{* * *} P<0.001$. 
Table 1. The correlation of NAP1L1 or HDGF protein expression with clinicopathological parameters in gliomas.

\begin{tabular}{|c|c|c|c|c|c|c|c|}
\hline \multirow{2}{*}{ Characteristics } & \multirow{2}{*}{$N$} & \multicolumn{2}{|c|}{ NAP1L1 } & \multirow{2}{*}{$P$ value } & \multicolumn{2}{|c|}{ HDGF } & \multirow{2}{*}{$P$ value } \\
\hline & & Low & High & & Low & High & \\
\hline \multicolumn{8}{|l|}{ Age (years) } \\
\hline$<55$ & 86 & 29 & 57 & 0.866 & 36 & 50 & 0.936 \\
\hline$\geq 55$ & 22 & 7 & 15 & & 9 & 13 & \\
\hline \multicolumn{8}{|l|}{ Gender } \\
\hline Male & 59 & 21 & 38 & 0.585 & 27 & 32 & 0.343 \\
\hline Female & 49 & 15 & 34 & & 18 & 31 & \\
\hline \multicolumn{8}{|l|}{ WHO grade } \\
\hline I & 7 & 6 & 1 & 0.013 & 6 & 1 & 0.026 \\
\hline II & 43 & 15 & 28 & & 21 & 22 & \\
\hline III & 18 & 6 & 12 & & 6 & 12 & \\
\hline IV & 40 & 9 & 31 & & 12 & 28 & \\
\hline \multicolumn{8}{|l|}{ Ki-67 index } \\
\hline$\geq 20 \%$ & 60 & 12 & 48 & 0.001 & 19 & 41 & 0.018 \\
\hline$<20 \%$ & 48 & 24 & 24 & & 26 & 22 & \\
\hline \multicolumn{8}{|l|}{ KPS } \\
\hline$\geq 80$ & 84 & 32 & 52 & 0.050 & 40 & 44 & 0.019 \\
\hline$<80$ & 24 & 4 & 20 & & 5 & 19 & \\
\hline \multicolumn{8}{|l|}{ Vital status } \\
\hline Alive & 45 & 21 & 24 & 0.013 & 26 & 19 & 0.004 \\
\hline Dead & 63 & 15 & 48 & & 19 & 44 & \\
\hline \multicolumn{8}{|l|}{ Recurrence } \\
\hline No & 23 & 16 & 7 & $<0.001$ & 16 & 7 & 0.002 \\
\hline Yes & 85 & 20 & 65 & & 29 & 56 & \\
\hline
\end{tabular}

Table 2. Univariate analysis of factors associated with OS and DFS in 108 glioma patients.

\begin{tabular}{lccccccc}
\hline \multirow{2}{*}{ Factors } & \multicolumn{3}{c}{ OS } & \multirow{2}{*}{ PR } & $\mathbf{9 5 \%} \mathbf{C I}$ & & \multicolumn{3}{c}{ DFS } & \multirow{2}{*}{$\boldsymbol{P}$-value } \\
\cline { 2 - 3 } & Hge & 0.536 & $0.302-0.951$ & $\mathbf{0 . 0 3 3}$ & 0.665 & $0.393-1.126$ & 0.129 \\
Gender & 1.232 & $0.750-2.022$ & 0.410 & 1.042 & $0.676-1.604$ & 0.853 \\
WHO grade & 1.868 & $1.425-2.448$ & $<\mathbf{0 . 0 0 1}$ & 1.438 & $1.162-1.780$ & $\mathbf{0 . 0 0 1}$ \\
Ki-67 index & 0.336 & $0.193-0.585$ & $\mathbf{< 0 . 0 0 1}$ & 0.400 & $0.252-0.633$ & $<\mathbf{0 . 0 0 1}$ \\
KPS score & 4.257 & $2.449-7.398$ & $\mathbf{0 . 0 0 1}$ & 3.468 & $2.060-5.841$ & $<\mathbf{0 . 0 0 1}$ \\
HDGF & 2.213 & $1.288-3.802$ & $\mathbf{0 . 0 0 4}$ & 2.174 & $1.371-3.446$ & $\mathbf{0 . 0 0 1}$ \\
NAP1L1 & 2.081 & $1.164-3.723$ & $\mathbf{0 . 0 1 3}$ & 2.399 & $1.443-3.989$ & $\mathbf{0 . 0 0 1}$
\end{tabular}

Table 3. Multivariate analysis of factors associated with OS and DFS in 108 glioma patients.

\begin{tabular}{lccccccc}
\hline \multirow{2}{*}{ Factors } & \multicolumn{2}{c}{ OS } & \multirow{2}{*}{ P-value } & \multicolumn{3}{c}{ DFS } & \multirow{2}{*}{$\boldsymbol{P}$-value } \\
\cline { 2 - 3 } & HR & $\mathbf{9 5 \%}$ CI & & HR & 95\% CI & \\
WHe & 0.574 & $0.317-1.040$ & 0.067 & & & & \\
WHO grade & 1.445 & $1.040-2.006$ & $\mathbf{0 . 0 2 8}$ & 1.096 & $0.839-1.431$ & 0.503 \\
Ki-67 index & 0.688 & $0.347-1.366$ & 0.286 & 0.671 & $0.353-1.081$ & 0.091 \\
KPS score & 2.393 & $1.306-4.386$ & $\mathbf{0 . 0 0 5}$ & 2.341 & $1.334-4.110$ & $\mathbf{0 . 0 0 3}$ \\
NAP1L1 & 1.372 & $0.740-1.040$ & 0.315 & 1.768 & $1.035-3.020$ & $\mathbf{0 . 0 3 7}$ \\
\hline
\end{tabular}


NAP1L1 controls the expression of genes associated with cell cycle and apoptosis through the CCND1/CDK4/CDK6 signaling pathway in glioma

To further study the mechanism underlying NAP1L1silencing mediated reduction in cell proliferation, we performed EdU analysis. The findings showed that NAP1L1 knockdown significantly inhibited the proliferation of glioma cells in comparison with that in the control group (Figure 3A). Cell cycle analysis showed that the suppression of NAP1L1 could significantly reduce the cell cycle progression from G1
A

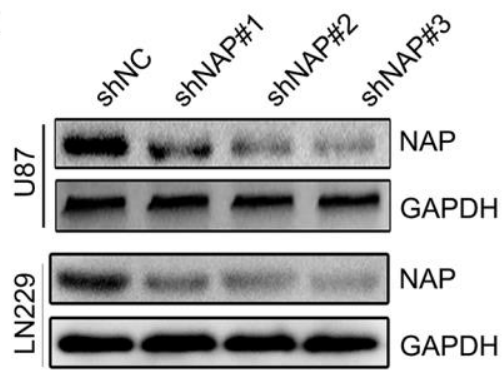

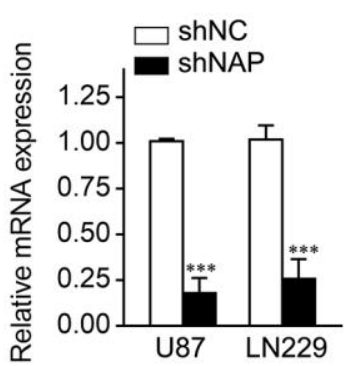

B

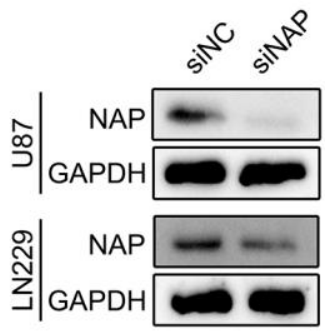

C
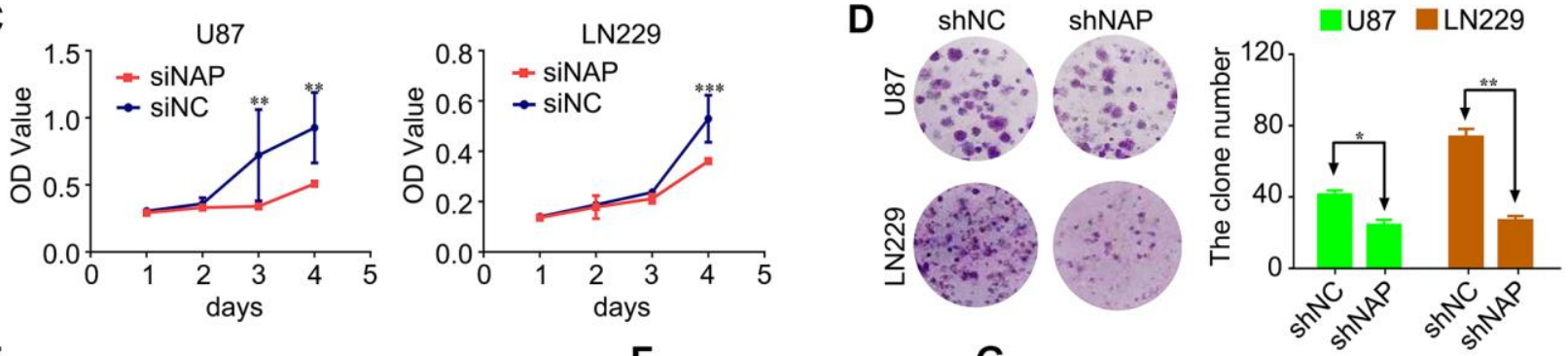

$\mathbf{E}$

$\mathbf{F}$
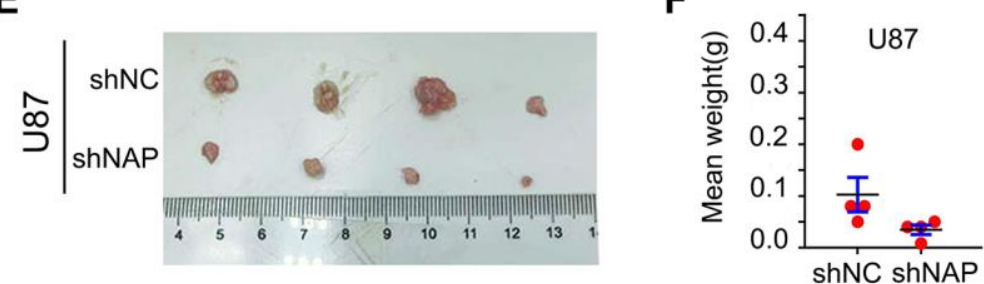

G
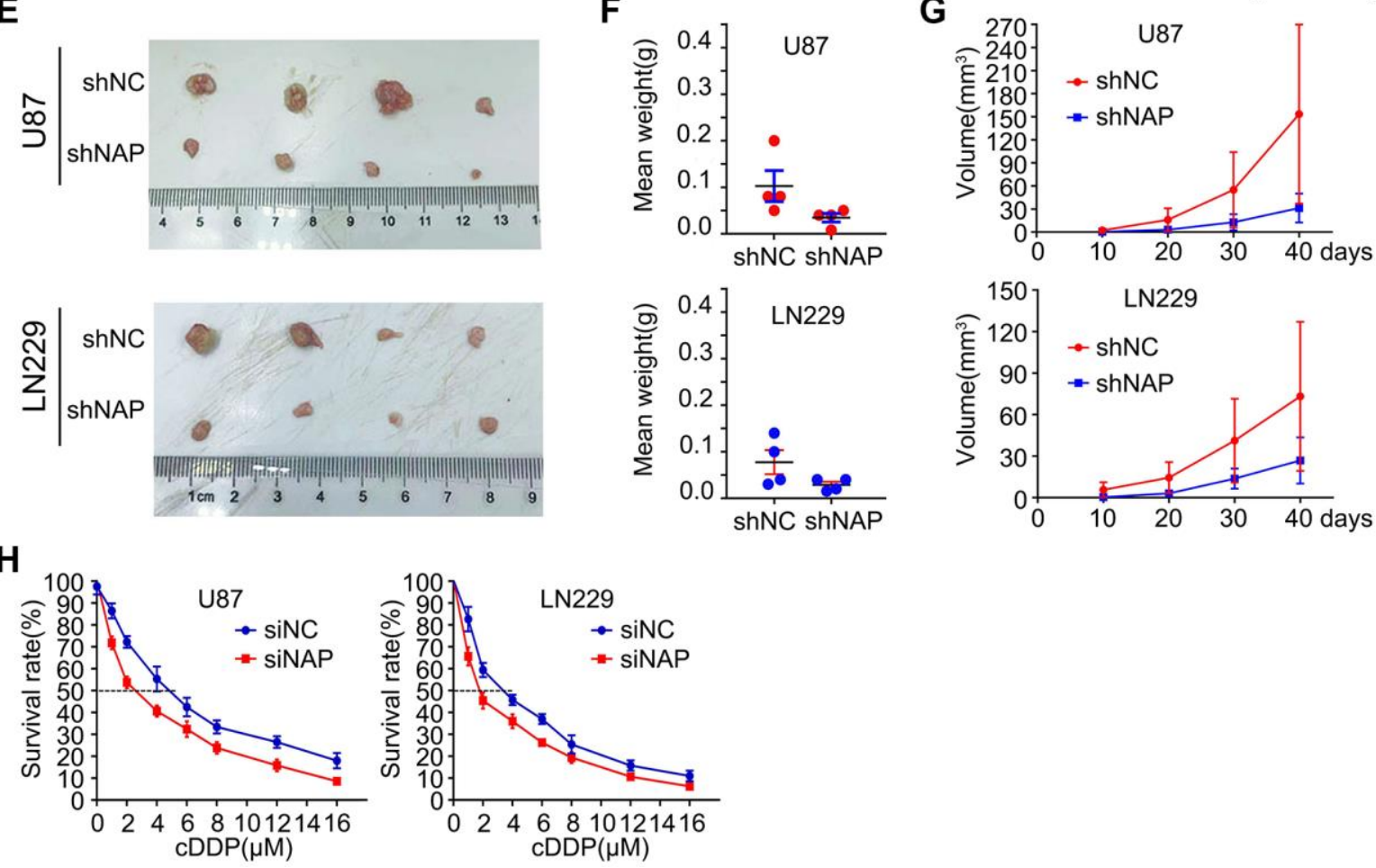

Figure 2. Suppressing NAP1L1 inhibits glioma cell proliferation and chemoresistance. (A) NAP protein level was measured by western blot in U87 and LN229 cells transfected with shNC or shNAP. GAPDH was used as a loading control. With efficient knockdowns from shNAP was separately detected by RT-PCR assays. (B) NAP protein level was measured by western blot in U87 and LN229 cells transfected with siNC or siNAP. GAPDH was used as a loading control. MTT assay (C), clone formation assay (D) after NAP1L1 knockdown. Gross morphology of tumors (E) and tumor weight statistics (F) from the indicated groups ( $n=4$ per group). (G) Tumor volume statistics for each mouse group ( $n=4$ per group). (H) Dose-response curves of U87 and LN229 treated with shNAP and shNC respectively following treatment with DDP for $48 \mathrm{~h}$. Data are presented as the mean \pm SD for three independent experiments. ${ }^{*} P<0.05,{ }^{* *} P<0.01,{ }^{* * *} P<0.001$. 
to $\mathrm{S}$ phase (Figure 3B). The cell apoptosis assay and mitochondrial membrane potential assay indicated that siNAP1L1 dramatically induced apoptosis in glioma cells (Figure 3C, 3D). Interestingly, glioma cells with stably silenced NAP1L1 also significantly inhibited the proliferation. The results were showed in Supplementary Figure 2A-2D. In addition, we investigated the protein levels of genes associated with cell cycle and apoptosis in the U251 and U87 glioma cells with stable suppression of NAP1L1. Western blotting demonstrated that siNAP1L1 inhibited the activation of oncogenic cell cycle regulators, such as CDK4, CDK6, and CCND1 (Figure 3E). Furthermore, a potent anti-apoptotic regulatory factor of $\mathrm{Bcl}-2$ significantly decreased in the U251 and U87 cells after transfection with siNAP1L1. As well, the protein level of cleaved caspase- 3 was upregulated in both the cell lines with stably suppressed NAP1L1 (Figure 3E).
A
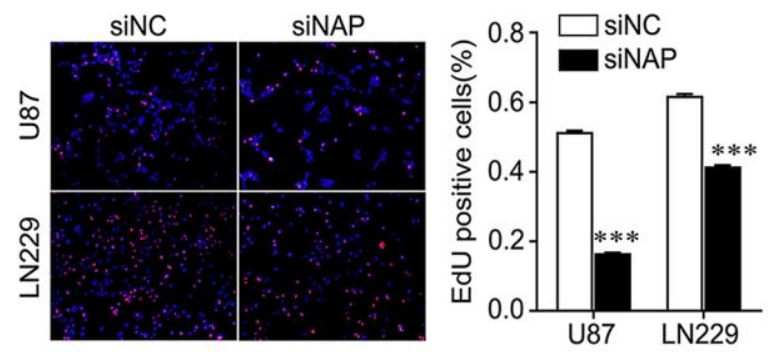

C
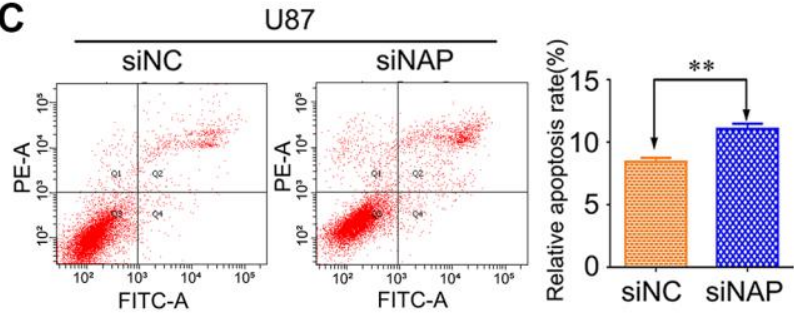

D
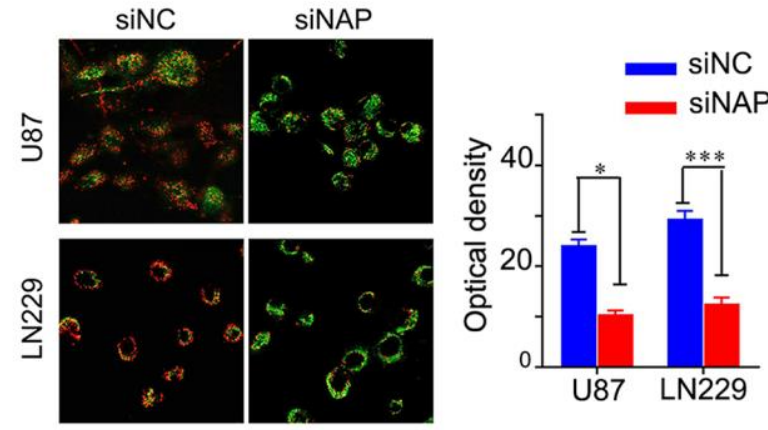

$\mathbf{F}$

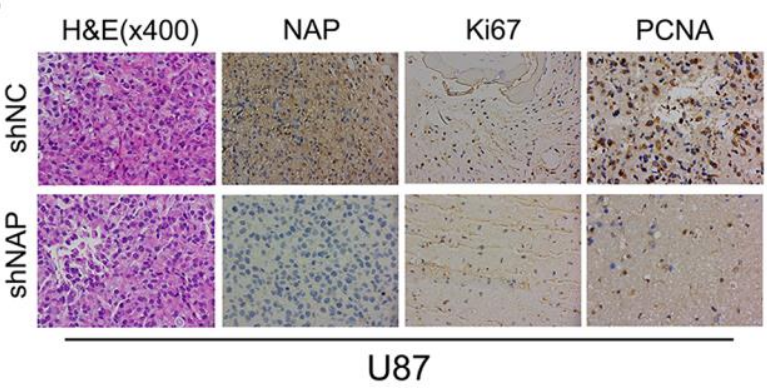

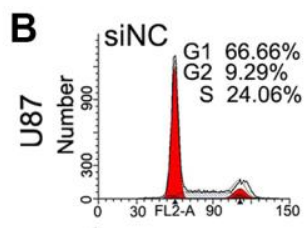
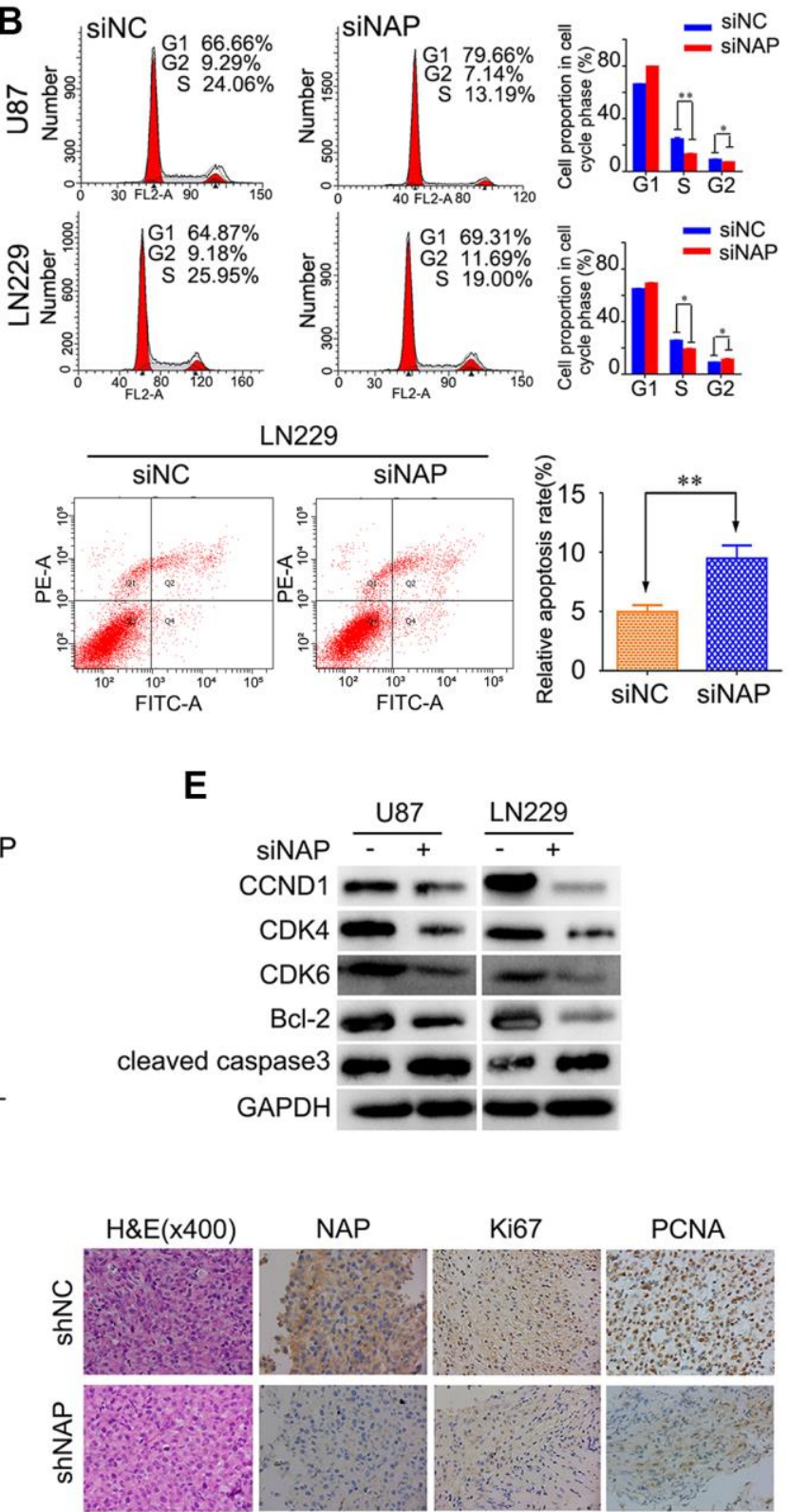

LN229

Figure 3. NAP1L1 controls the expression of cell cycle and apoptosis associated genes via the CCND1/CDK4/CDK6 signaling pathways in glioma. EdU incorporation assay (A), Cell cycle analysis (B), Cell apoptosis assay (C) Mitochondrial membrane potential assay (D) in U87 and LN229 cells transfected with control siRNA (siNC) or NAP1L1 siRNA (siNAP). (E) Western blotting analysis of the protein levels of CCND1, CDK4, CDK6, Bcl-2 and cleaved caspase3 after transfecting siNC or siNAP into U87 and LN229 cells. (F) The H\&E of the nude mice tumor tissues. NAP1L1, Ki67, and PCNA was evaluated by immunohistochemical staining. Compared with shNAP cells, the shNC cell tumor tissues were high expression. ${ }^{*} P<0.05,{ }^{* *} P<0.01,{ }^{* * *} P<0.001$. 
Moreover, the mice injected with shNAP1L1-U87 and shNAP1L1-LN229 cells exhibited lower expressions of Ki67, proliferating cell nuclear antigen (PCNA) and NAP1L1 in tumor tissues relative to the control mice (Figure 3F). These data demonstrated that NAP1L1 could dramatically promote cell proliferation through the CDK4/CDK6/CCND1 signaling axis in glioma.

\section{NAP1L1 interacts with HDGF and HDGF knockdown reverses the proliferative effect in glioma cells overexpressing NAP1L1}

In our preliminary work, using mass spectrometry, we predicted a direct interaction between NAP1L1 and HDGF proteins, and this was confirmed in endometrial carcinoma (unpublished data). Thus, coimmunoprecipitation (co-IP) was performed to examine the relevant binding partners of NAP1L1. Endogenous co-IP assay indicated an interaction between HDGF and NAP1L1 in the U251 and U87 cells (Figure 4A). Immunofluorescence assay confirmed their colocalization in the cytoplasm of glioma cells (Figure 4B). In addition, NAP1L1 overexpression could upregulate HDGF expression in glioma cells, while NAP1L1 knockdown decreased the protein level expression of HDGF (Figure 4C) (Supplementary Figure 2E). To further evaluate the relationship between HDGF and NAP1L1, we performed MTT and EdU assays using NAP1L1-overexpressing glioma cells upon HDGF knockdown (Figure 4D, 4E). The results indicated that HDGF knockdown could significantly reverse NAP1L1 overexpression-mediated enhanced cell proliferation; the findings from western blotting further confirmed this result (Figure 4F). These results demonstrated that NAP1L1 interacted with HDGF, and further, induced cell proliferation in glioma.

\section{HDGF interacts with c-Jun and c-Jun overexpression reverses the proliferative effect upon HDGF knockdown in glioma cells}

To further clarify the mechanism of NAP1L1-mediated enhanced cell proliferation through HDGF in glioma cells, The BIOGRID database was used to identify the proteins that could interact with HDGF. We predicted that HDGF and c-Jun would interact directly. Thus, co-IP was completed to examine the relevant binding partners of HDGF. The endogenous co-IP assay verified the interaction between HDGF and c-Jun in the U251 and U87 cells (Figure 5A). Immunofluorescence assay showed a strong co-localization signal mainly in the cytoplasm; minor nuclear distribution was also observed (Figure 5B). Moreover, HDGF overexpression could upregulate c-Jun expression in glioma cells, while HDGF knockdown attenuated c-Jun expression at the protein level (Figure 5C) (Supplementary Figure 2F). To further evaluate the relationship between HDGF and c-Jun, MTT assay and EdU assays were completed to examine the reversal of the effect of c-Jun on HDGF knockdown in glioma cell proliferation (Figure 5D, 5E). Western blotting illustrated that c-Jun/CCND1/CDK4/CDK6 signaling was dramatically enhanced (Figure 5F). Taken together, these results demonstrated that c-Jun enhanced upon HDGF overexpression could promote glioma cell proliferation by inducing the activation of the CCND1/CDK4/CDK6 signaling axis.

\section{Correlation of NAP1L1 and HDGF expression with overall survival in patients with glioma}

To determine the role of NAP1L1 and HDGF in glioma, we analysed the protein level expressions of NAP1L1 and HDGF by immunostaining in 108 glioma tissues and 24 para-tumor tissues (Figure 6A). Immunohistochemical (IHC) staining for the expressions of NAP1L1 and HDGF showed that NAP1L1 positive signals were mostly localized to the cytoplasm of glioma cells, while those of HDGF was mainly in the cytoplasm and nuclei (Figure 6A). In addition, IHC analysis demonstrated that tumors having high expression of NAP1L1 also had significantly higher expression of HDGF as compared to those with low expression of NAP1L1 (Figure 6B). Next, the prognostic implications of NAP1L1 and HDGF in gliomas were assessed. Kaplan-Meier survival analysis illustrated that patients with high expression levels of HDGF had dramatically shortened OS times (Figure 6C) compared with patients with low level of HDGF expression. Interestingly, patients with both high HDGF and NAP1L1 expressions had the shortest survival duration (Figure 6D).

To verify the functions of NAP1L1 and HDGF, we analyzed the correlation of NAP1L1 and HDGF expressions with widely recognized clinicopathological parameters in glioma specimens. As shown in Table 1, high HDGF expressions in gliomas were significantly associated with WHO grades II IV, KPS $<80$, Ki-67 index $\geq 20 \%$, and recurrence. Furthermore, univariate analysis showed that the WHO grade, KPS, Ki-67 index, recurrence, NAP1L1 and HDGF expressions were unfavorable predictors for overall survival (Table 2). Correlation analysis indicated a significantly positive correlation between NAP1L1 and HDGF expressions in glioma tissues (Table 4, $\mathrm{r}=0.279, P=$ $0.003)$.

\section{DISCUSSION}

Despite huge progress in the development of diagnostic and therapeutic methods, including radiation treatment 
and chemotherapy, glioma remains one of the most lethal cancers in humans $[10,11]$. The average survival period of patients with glioma is less than two years and the five-year survival rate is less than $3 \%$, the lowest among all cancers [12]. Thus, it is important to develop novel diagnostic methods and effective treatment strategies. NAP1L1 is the human analog of the yeast NAP-I protein, a histone-binding factor involved in the maintenance of cumulative nucleosome formation [13]. In humans, NAP1L1 belongs to a family of proteins involved in nucleosome assembly and transcriptional regulation [14]. Both mRNA and protein levels of NAP1L1 increase rapidly in conjunction with cell proliferation in a T-lymphoid cell model [15]. NAP1L1 is upregulated in small-intestinal neuroendocrine cellderived neoplastic tissues [16], colon cancer [17], and
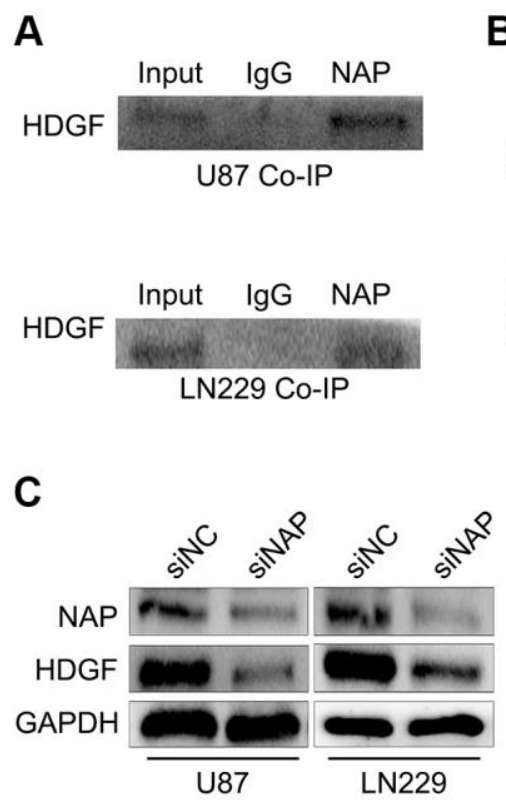

$\mathbf{E}$

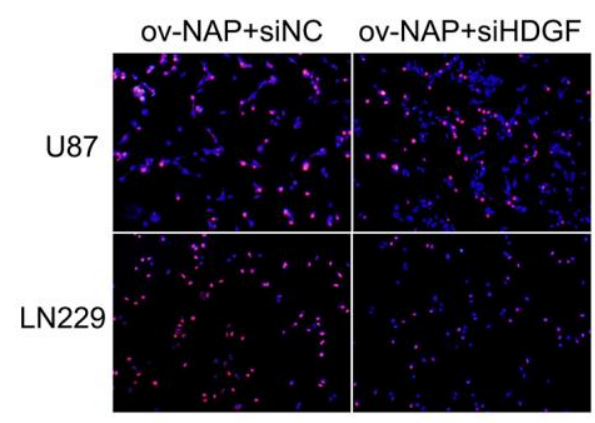

F

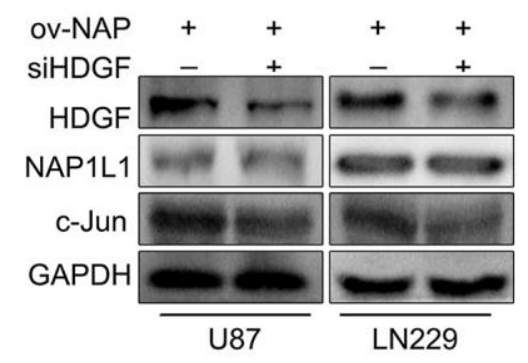

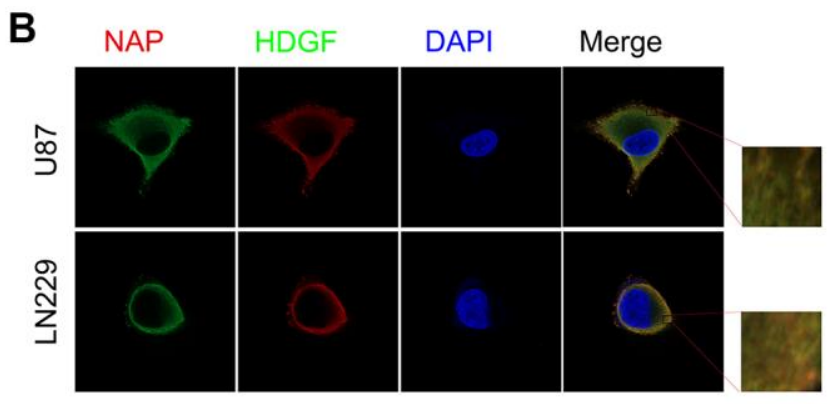

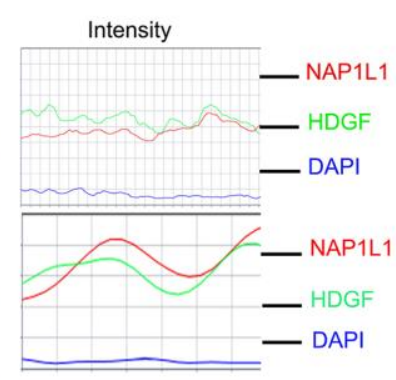

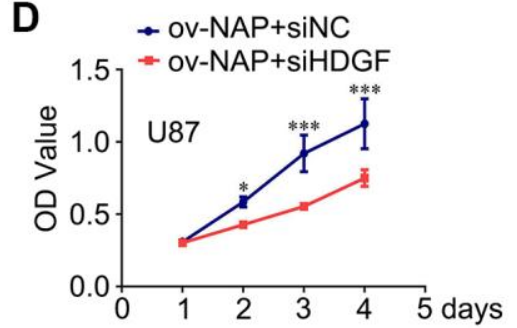

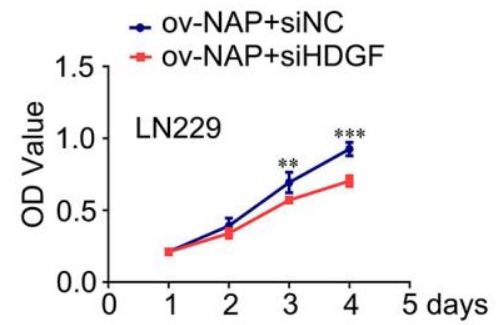

Figure 4. NAP1L1 interacts with HDGF and HDGF knockdown reverses the effect of overexpressed NAP1L1 on proliferation of glioma cells. (A) Co-IP experiments detected the interaction of endogenous NAP1L1 and HDGF in U87 and LN229 cells. (B) Representative immunofluorescence staining and intensity of NAP1L1 and HDGF protein in U87 and LN229 cells. Scale bar, $5 \mu \mathrm{m}$. (C) HDGF level in U87 and LN229 cells transfected with siNAP1L1 or NAP1L1-overexpressing plasmid. MTT assay (D) and EdU incorporation assay (E) in glioma cells transfected with control or HDGF siRNA. (F) Western blotting analysis of the protein levels of HDGF, NAP1L1 and c-Jun after transfection of siHDGF into glioma cells. GAPDH served as the internal control. Data are presented as the mean \pm SD for three independent experiments. ${ }^{*} P<0.05,{ }^{* *} P<0.01,{ }^{* * *} P<0.001$. 
malignant adenocarcinoma as compared to normal mucosa [18]. NAP1L1 is involved in the ovarian cancer cell response to cytotoxic gold compounds [19]. In the last two decades, extensive studies show that NAP1L1 is an important regulator, critical for various biological processes in multiple cancer types [5, 8]. However, its role in human glioma remains unknown.

To address the problem, first, we analysed the mRNA level of NAP1L1 in the TCGA database samples. The data demonstrated that at the mRNA level, the expression of NAP1L1 was elevated in glioma. Furthermore, OS and DFS analyses illustrated that NAP1L1 overexpression was an unfavorable factor associated with reduced survival time of glioma patients. These results indicated that NAP1L1 is an important putative oncogene in glioma. The RT-qPCR analysis also confirmed that the mRNA level of NAP1L1 was dramatically upregulated in 24 gliomas tissues as compared to the corresponding 24 para-tumor
A

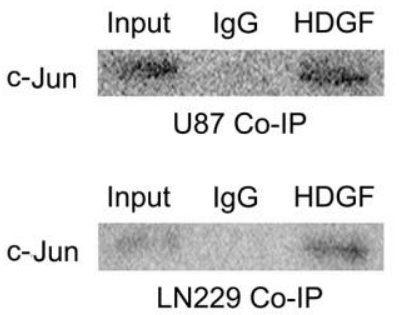

C

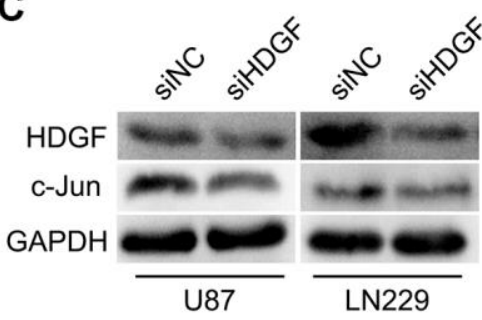

D

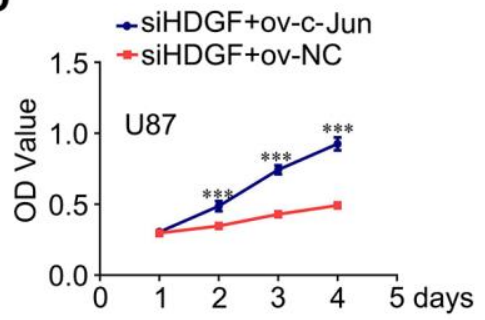

B

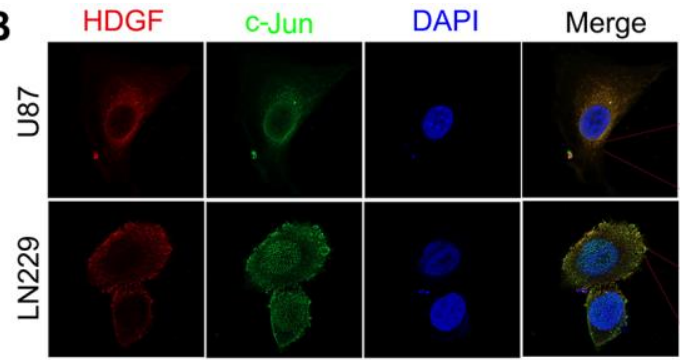

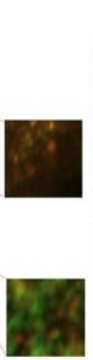

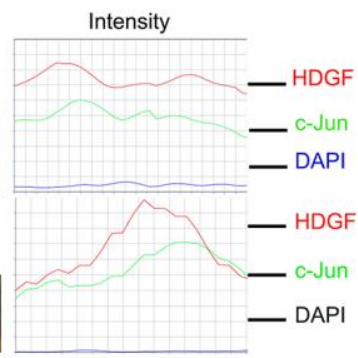

E
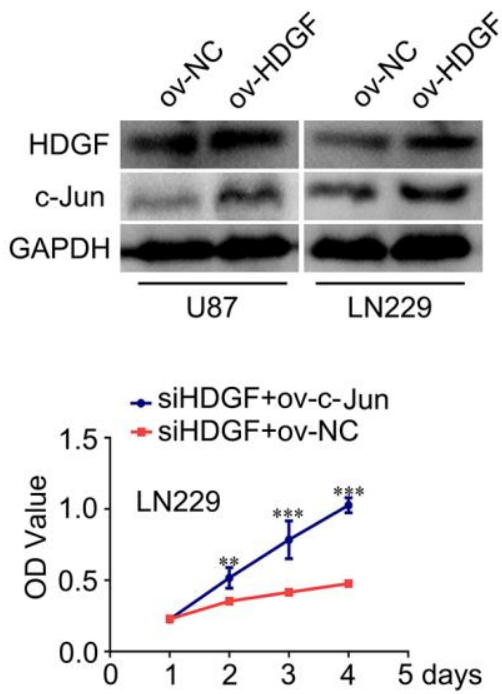
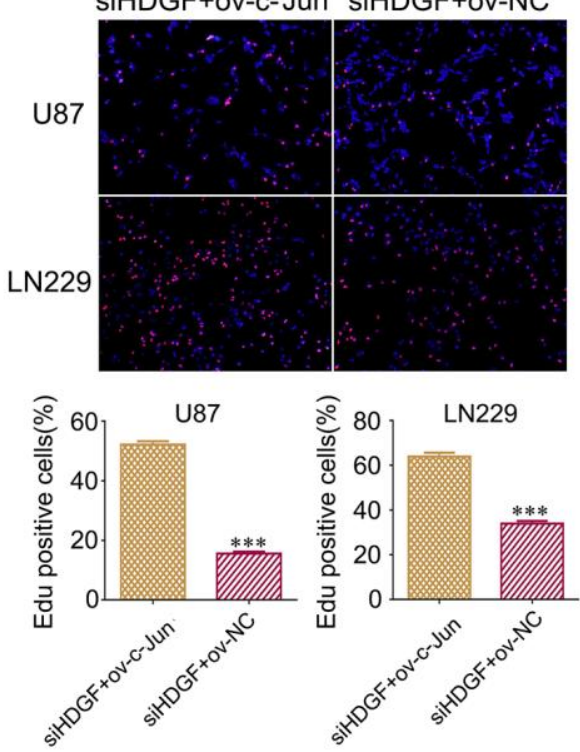

$\mathbf{F}$

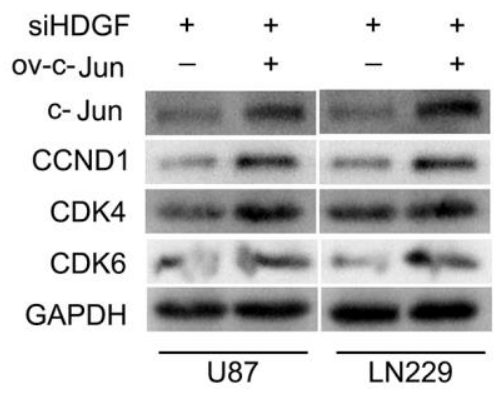

Figure 5. HDGF interacts with c-Jun and C-Jun overexpression reverses the effect of HDGF knockdown on proliferation of glioma cells. (A) Co-IP experiments detected the interaction of endogenous HDGF and c-Jun in U87 and LN229 cells. (B) Representative immunofluorescence staining and intensity of HDGF and c-Jun protein in U87 and LN229 cells. Scale bar, $5 \mu \mathrm{m}$. (C) c-Jun expression in U87 and LN229 cells transfected with siHDGF or HDGF-overexpressing plasmid. The MTT assay (D) and EdU incorporation assay (E) in glioma cells after transfecting c-Jun-overexpressing plasmid. (F) Western blotting for c-Jun, CCND1, CDK4 and CDK6 in glioma cells after transfected with c-Jun-overexpressing plasmid. GAPDH served as the internal control. Data are presented as the mean \pm SD for three independent experiments. ${ }^{*} P<0.05,{ }^{* *} P<0.01,{ }^{* * *} P<0.001$. 
tissues. A tissue microarray (TMA) containing 108 glioma and 24 para-tumor tissue samples was used to assess the NAP1L1 expression level. Next, we found that downregulated NAP1L1 attenuated the proliferative ability of glioma cells in vivo and in vitro. Furthermore, reducing the level of NAP1L1 enhanced the sensitivity to cDDP chemotherapy in glioma cells. Our results indicated that NAP1L1 may be a tumor promoter that could participate in glioma pathogenesis. Prior investigation from our group using mass spectrometry combined with exogenous co-IP, indicated that HDGF was a potential interacting partner of NAP1L1 in endometrial carcinoma (unpublished data). To better understand the underlying molecular mechanisms, we used endogenous co-IP and micro-confocal colocalization assays to confirm NAP1L1 binding with HDGF and their colocalization in the cytoplasm. The findings demonstrated that NAP1L1 interacted with HDGF in glioma.

HDGF is an important oncogene involved in proliferation, invasion, and metastasis in liver cancer, stomach cancer, prostate cancer, and non-small cell lung cancer [20-23]. Our previous study indicated that patients with glioma had a poor prognosis when expression of HDGF was abnormal [24]. These results demonstrated the significance of HDGF in gliomas pathogenesis. In this study, we verified that the mRNA levels of HDGF were increased in 24 gliomas as compared to the corresponding 24 para-tumor tissue samples (Supplementary Figure 2G). Consistent with the mRNA levels, expression of the HDGF protein was dramatically upregulated in glioma. We also found that the expression of HDGF was positively correlated with the tumor WHO grade, as shown by the dramatical differences between high- and low-grade glioma patients. Nonetheless, the specific details of HDGFinduced proliferation of glioma remain unexplored. To examine the HDGF-mediated promotion of glioma cell proliferation, the BIOGRID database was used to predict the interacting protein partners of HDGF, and c-Jun was identified as a potential candidate. Indeed, the interaction of HDGF and c-Jun in glioma cells was confirmed by endogenous co-IP assay. Immunofluorescence analyses illustrated that c-Jun and HDGF proteins mostly co-localized in the cytoplasm
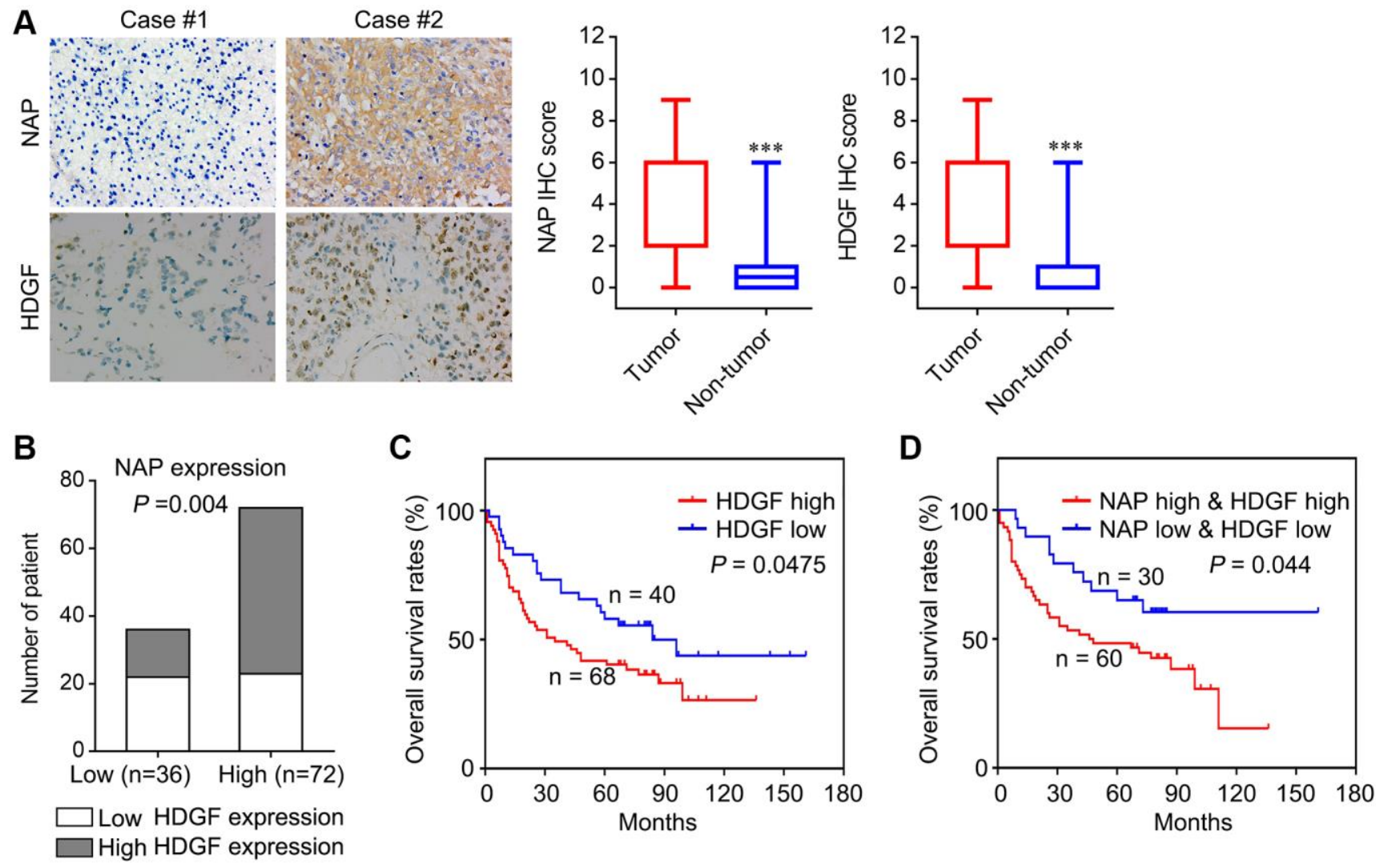

Figure 6. Correlation analysis of NAP1L1 and HDGF with overall survival of patients with glioma. (A) Representative immunohistochemistry images and IHC score of expression of NAP1L1 and HDGF in glioma tissues, respectively. Original magnification 400x. (B) Correlation analysis of NAP1L1 and HDGF with overall survival of patients with glioma. Prognostic significance assessed by Kaplan-Meier survival estimates. Comparison of the overall survival by HDGF (C), respectively. (D) Kaplan-Meier analysis of overall survival in patients with variable expression of NAP1L1 and HDGF. ${ }^{*} P<0.05,{ }^{* *} P<0.01,{ }^{* * *} P<0.001$. 
Table 4. Correlation between NAP1L1 and HDGF expression in glioma tissues.

\begin{tabular}{llccccc}
\hline & & \multicolumn{2}{c}{ NAP1L1 expression } & & \multirow{2}{*}{-Value } & \multirow{2}{*}{$\boldsymbol{P}$-Value } \\
\cline { 3 - 4 } & & Low & High & & \multirow{2}{*}{0.003} \\
\hline \multirow{2}{*}{ HDGF expression } & Low & 22 & 23 & 0.279 & 0.003 \\
& High & 14 & 49 & & \\
\hline
\end{tabular}

of glioma cells; minor nuclear distribution was also observed. In addition, knocking down HDGF significantly decreased the expressions of c-Jun/ CCND1/CDK4/CDK6 at the protein level. c-Jun is an oncogenic transcription factor, which can modulate the cell cycle and other tumor pathogeneses mechanisms by transcription or suppression of gene expression [25, 26]. Previous studies show that c-Jun regulates cell proliferation in non-small cell lung cancer by targeting CCND1 [27]. CCND1 is a transcription product of cJun [28]; it is a cell cycle promoter inducing cell proliferation in tumors [29]. CDK4/CDK6 is activated by CCND1. Next, western blotting was performed to evaluate the reversal upon c-Jun-overexpression on glioma cell proliferation due to HDGF knockdown. Upregulated CCND1/CDK4/CDK6 expressions were confirmed. Taken together, these results demonstrated that HDGF-mediated c-Jun regulation could promote glioma cell proliferation by inducing CCND1/CDK4/ CDK6.

Consistent with their previously described roles in glioma cells, we performed the immunohistochemistry assay to examine NAP1L1 and HDGF expressions in 108 gliomas tissues compared to those in 24 para-tumor tissues. High expression of NAP1L1 was positively correlated with WHO grade, KPS, Ki-67 index, and recurrence, which indicated that NAP1L1 could promote glioma progression. Similarly, high expression of HDGF was positively correlated with WHO grade, KPS, Ki-67 index, and recurrence, which suggested that HDGF was also involved in glioma progression. Patients with high NAP1L1 or HDGF expression levels had significantly shortened OS times as indicated by the Kaplan-Meier analysis. Moreover, NAP1L1 expression was positively correlated with the expression of HDGF in glioma tissues. Glioma patients having high expression of both NAP1L1 and HDGF showed the worst survival prognoses as compared to other groups. These data further confirmed that enhanced NAP1L1 and HDGF expressions were important factors that synergistically promoted the development and spread of glioma.

As shown in Figure 7, a regulatory model is illustrated. NAP1L1 interacts with HDGF, and the latter recruits

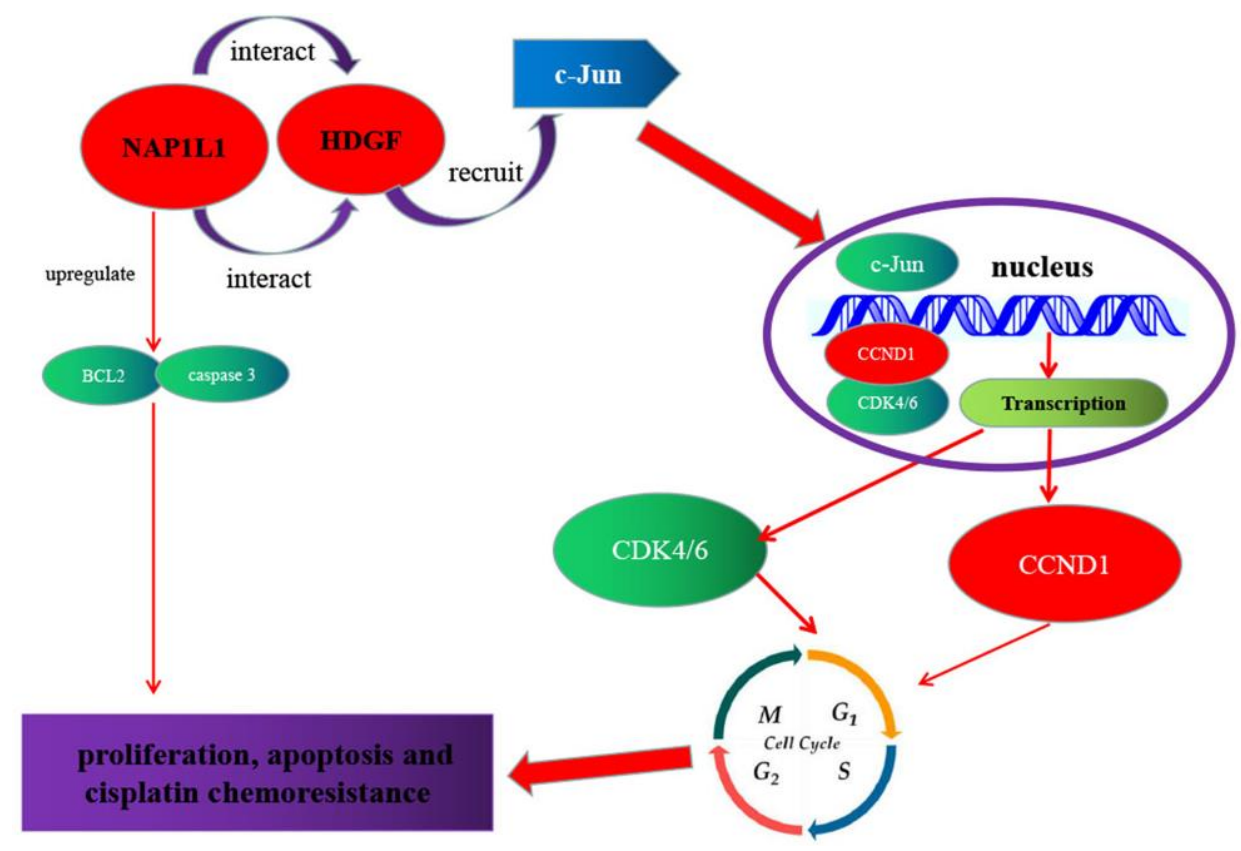

Figure 7. Schematic of NAP1L1 promoting glioma development. NAP1L1 interacts with HDGF, while the latter recruits c-Jun, a key oncogenic transcription factor that can induce CCND1/CDK4/CDK6 expression, and thus promotes proliferation and chemoresistance of glioma cells. 
c-Jun, a key oncogenic transcription factor, thereby inducing CCND1/CDK4/CDK6 expressions and promoting proliferation and chemoresistance in glioma cells. The high expressions of NAP1L1 or/and HDGF in glioma tissues indicate shorter overall survival in glioma patients.

In summary, NAP1L1 could promote proliferation and chemoresistance in glioma cells by interacting with HDGF and activating c-Jun to induce the expressions of CCND1/CDK4/CDK6. Thus, NAP1L1 has the potential to be a promising biomarker as well as therapeutic target for the pathogenesis of glioma. In the future, more studies are required to further evaluate the functions of NAP1L1.

\section{MATERIALS AND METHODS}

\section{Cells and patients}

Human glioma cell lines U87 and LN229 were obtained from the Cancer Research Institute of Southern Medical University and cultured in DMEM medium containing $10 \%$ fetal bovine serum (ExCell, Uruguay) at $37^{\circ} \mathrm{C}$ with $5 \% \mathrm{CO}_{2}$. Moreover, glioma and para-tumor tissues were obtained from patients undergoing a surgical procedure at the Nanfang Hospital, Southern Medical University. Ethics approval from the Ethics Committee of the Nanfang Hospital and patient consent were obtained in this study.

\section{Lentivirus production and infection}

Lentiviral vectors harboring shRNA-targeting NAP1L1 and negative control vectors were both established by GeneChem (Shanghai, China). U87 and LN229 cell lines stably expressing shRNA or negative control (NC) vectors were performed as described in a previous study. The NAP1L1 shRNA sequence are presented in Supplementary Table 1. Infection efficiency was showed by green fluorescent protein ratio. The expression of shNAP1L1 was measured by RT-qPCR.

\section{Transient transfection using plasmids or small interfering RNAs}

NAP1L1 and c-Jun plasmids were generated by Guangzhou IGE (IGE Inc., China). Small interfering RNA (siRNA) for NAP1L1, HDGF (named as siNAP and siHDGF, respectively) and the control sequences (siNC) were designed by Guangzhou RiboBio (RiboBio Inc., China). The siNAP1L1, siHDGF sequences are presented in Supplementary Table 2. According to the manufacturer's protocol, siRNA or plasmids were transfected into U87 and LN229 cells using
Lipofectamine ${ }^{\circledR} 3000$ (Invitrogen; Thermo Fisher Scientific, Inc., Waltham, MA, USA). Cells were collected after 48-72 $\mathrm{h}$ for further experiments.

\section{Reverse transcription-quantitative polymerase chain reaction (RT-qPCR)}

Total mRNA was obtained from clinical fresh tissues or cultured cells using QIAZOL (Qiagen, Shanghai, China). And $1 \mu \mathrm{g}$ extracted mRNA was used for Complementary DNA (cDNA) synthesis with random primers and Maxima First Strand cDNA Synthesis Kit (Takara Bio, Inc., Otsu, Japan). Real-time quantitative PCR (RT-qPCR) was performed according to the manufacturer's instructions using the SYBR Green Master Mix (TAKARA) and LightCycler480II system (Roche). GAPDH used as the reference gene for NAP1L1 or HDGF or c-Jun; and the relative expression of RNAs was calculated using formula [30]. The primers were listed in the Supplementary Table 3.

\section{MTT assay}

MTT assay was used to perform drug sensitivity tests and cell proliferation. This assay was conducted as described previously [31]. In brief, about 2,000 glioma cells were seeded per well in 96-well plates. After cell adherence, the cells were incubated with MTT at $37^{\circ} \mathrm{C}$ for $4 \mathrm{~h}$ to produce formazan. Then the formazan crystals formed by viable cells were solubilized in $150 \mu 1$ dimethyl sulfoxide (Sigma). Finally, the absorbance value (OD) was measured at $490 \mathrm{~nm}$ by Universal Microplate Reader (Bio-Tek instruments, Inc., Winooski, VT, USA).

\section{EdU analysis}

The Cell-Light EDU Apollo 488 or 567 in vitro Imaging kit (Guangzhou Ribobio Co., Ltd.) was used to measure glioma cells proliferation, according to the manufacturer's introduction. Briefly, following incubation with $10 \mathrm{mM}$ EdU for $2 \mathrm{~h}$ at $37^{\circ} \mathrm{C}$ with $5 \%$ $\mathrm{CO}_{2}$, the U87 and LN229 cells were fixed with $4 \%$ paraformaldehyde, permeabilized with Triton X-100 $(0.2 \%)$, and stained with Apollo fluorescent dyes and costained with $5 \mu \mathrm{g} / \mathrm{mL}$ DAPI. Finally, EdU-positive cells were counted under a fluorescence microscope in five random fields. All experiments were independently performed at least three times.

\section{Clone formation assays}

U87 and LN229 cells were plated in six-well plates at a density of 1,000 per well, followed by incubation at $37^{\circ} \mathrm{C}$ in $5 \% \mathrm{CO}_{2}$ for two weeks. The colonies were 
fixed with $0.4 \%$ gluteraldehyde and stained with $0.1 \%$ crystal violet for $15 \mathrm{~min}$. Then the number of colonies containing $\geq 50$ cells was counted under a microscope. This assay was performed as described previously [32].

\section{Cell apoptosis and cell cycle distribution analysis}

Cell apoptosis and cell cycle distribution of the indicated cells were respectively determined using the cell cycle detection kit (BD Biosciences, San Jose, CA) or Annexin V Apoptosis Detection Kit (BD), according to the manufacturer's instructions by a BD LSRFortessa X-20 (BD). The data were analyzed using FlowJo software version 10.4 (Tree Star, Inc., San Carlos, CA).

\section{Mitochondrial membrane potential assay}

The U87 and LN229 cells were seeded in confocal culture dishes and cultured during the night. In brief, according to the manufacturer's introduction (Yitabio, Beijing, China), U87 and LN229 cells were stained with JC-10. Then use a Carl Zeiss LSM800 confocal laser scanning microscope to test the samples. The Fiji software is used for the statistical analysis of optical density [33].

\section{Immunofluorescence and confocal microscopy}

The U87 and LN229 cells were separated and seeded on a $35 \mathrm{~mm}$ glass bottom cell culture dish (SORFA, ZJ, China) at a density of 4000 cells/well. After cell adherence, the U87 and LN229 cells were fixed with paraformaldehyde (4\%) and permeabilized in $0.2 \%$ Triton X-100. Then the cells were incubated with specific antibodies (The antibodies are presented in Supplementary Table 4), counter stained with DAPI $(0.2 \mathrm{mg} / \mathrm{ml})$, and imaged using a Carl Zeiss LSM800 confocal laser scanning microscope.

\section{Co-immunoprecipitation (co-IP)}

The U87 and LN229 cells cultured in six-well plates were lysed with $600 \mu \mathrm{l}$ Pierce IP Lysis buffer containing protease and phosphatase inhibitor cocktails. According to the manufacturer's protocol, the Thermo Fisher Scientific Pierce co-IP kit was used to carry out co-IP. Total proteins were collected from cells and used for protein quantification. The specific anti-HDGF, NAP1L1, c-Jun and normal rabbit IgG (Supplementary Table 4) antibodies $(10 \mu \mathrm{g})$ was combined with $5 \mathrm{mg}$ protein to incubate overnight. The recovered proteins were analyzed by western blotting as described previously [34].

\section{Western blotting}

Protein was extracted from glioma cells using radioimmunoprecipitation assay buffer (Beyotime Institute of Biotechnology) containing PMSF (Bio-Rad Laboratories, Inc.) and Phosphatase inhibitors (Bio-Rad Laboratories, Inc.) (100:1:1). Then proteins were separated by SDS-PAGE gel electrophoresis and transferred to a PVDF membrane (Beyotime Institute of Biotechnology). Next, the indicated primary antibodies were used for immuno-detection with horseradish peroxidase-conjugated goat anti-rabbit or anti-mouse IgG antibodies and electrochemiluminescence chromogenic kit (Beyotime Institute of Biotechnology). The antibodies used for western blot were showed in the Supplementary Table 4 . The experiments were repeated at least three times.

\section{Animal studies in vivo}

The 4-week-old male nude mice (BALB/c, male) were purchased from the Experimental Animal Center of Southern Medical University (Guangzhou, China). The animals were maintained under a controlled temperature $\left(20 \pm 2^{\circ} \mathrm{C}\right)$ with 12 -h light/12-h dark cycles and ad libitum access to food and water. To study tumor growth, a subcutaneous xenograft mouse model was established. A total number of $2.0 \times 106$ U87 or LN229 cells were suspended in $100 \mu \mathrm{PBS}$ and subcutaneously injected into the flanks of 4-week-old male nude mice ( $N=5$ per group; left side, sh-NC; right side, shNAP). After 6 days, the tumors were measured using a caliper, and tumor volume was calculated as follows: $\mathrm{V}=\mathrm{L} \times$ $\mathrm{W} 2 \times 0.5236$, where $\mathrm{L}$ is the length and $\mathrm{W}$ is the width of the tumor. After 30 days, the tumors were harvested, weighed and photographed. All animal protocols were approved by the Institutional Animal Ethical Committee, Experimental Animal Center of Southern Medical University.

\section{Immunohistochemical staining (IHC)}

Paraffin sections of mouse tumors were used to assess the protein expression of NAP1L1, Ki67 and PCNA by immunohistochemistry assays. According to the manufacturer's introduction, the indirect streptavidinperoxidase method was used for immunohistochemistry assays. The assay was completed as previously described [35]. Immunohistochemical staining intensity was evaluated separately by two professional pathologists. A staining score of $\geq 6$ was classified as high NAP1L1 expression and a staining score of $\leq 4$ was considered to have low NAP1L1 expression. The antibodies are presented in Supplementary Table 4. 


\section{Database}

The RNA-seq V2 expression data and corresponding clinical information of TCGA CCA cohorts (163 tumor and 207 non-tumor samples) were attained from Gene Expression Profiling Interactive Analysis (GEPIA2) data portal (http://gepia2.cancer-pku.cn/\#index). According to the quartile cut point of the expression of NAP1L1, they were divided into 2 groups: weak expression of NAP1L1 group (the first quartile group, $n=41$ ) and strong expression of NAP1L1 group (the fourth quartile group, $n=41$ ), then the survival analysis was statistically analyzed.

\section{Statistical analysis}

Each experiment was performed with more than three times and data are expressed as the means \pm SD. Statistical significant difference was conducted using GraphPad Prism 9.1 (GraphPad Software, Inc.) and IBM SPSS Statistics version 23.0 (IBM SPSS, Inc., Chicago, IL, USA) software. One-way ANOVA or Student's two-tailed $t$ test was performed for comparison between groups. Statistical significance is indicated for each graph (n s, $P>0.05 ;{ }^{*} P<0.05 ;{ }^{* *} P<$ $\left.0.01 ;{ }^{* * *} P<0.001\right)$, which pointed to its potential role in this type of human malignancy.

\section{Availability of data and materials}

The datasets used and/or analyzed during the current study are available from the corresponding author on reasonable request.

\section{Ethics approval and consent to participate}

The Ethics Committee of The Integrated Hospital of Traditional Chinese Medicine, Southern Medical University authorized the experimental and research protocols of this study. All procedures performed in this study were according with the ethical standards of the institutional research committee and with the 1964 Helsinki declaration and its later amendments or comparable ethical standards. Written informed consent was provided and signed by all patients prior to sample collection. All animal experiments were conducted strictly according with the recommendations in the Guide for the Care and Use of Laboratory Animals of Southern Medical University.

\section{Abbreviations}

NAP1L1: nucleosome assembly protein 1 Like 1; HDGF: hepatoma-derived growth factor; TCGA: the cancer genome atlas; OS: overall survival; RT-qPCR: real time quantitative polymerase chain reaction; TMA: tissue microarray; DFS: disease free survival; H\&E: hematoxylin and eosin; KPS: Karnofsky Performance Status Scale.

\section{AUTHOR CONTRIBUTIONS}

Z.G.C and L.Y.L conceived of the study and supervised and coordinated all aspects of the work; X.Y.Y, H.C.L, Y.S, T.S.Q, R.T.H and H.T.H designed the research, wrote the paper, and prepared figures and tables; K.X.L, C.Y.L, C.J.Q, C.H.Z and H.L performed experiments, interpreted data, and prepared figures and tables; and W.Y.F and Q.S.L contributed analytical tools. All authors read and approved the manuscript.

\section{ACKNOWLEDGMENTS}

We gratefully thank all of the authors for this study.

\section{CONFLICTS OF INTEREST}

The authors declare no conflicts of interest related to this study.

\section{FUNDING}

This work was supported by the National Natural Science Foundation of China (grant no. 81760450), Natural Science Foundation of Guangxi Province (grant no. 2017GXNSFAA198322, 2019GXNSFDA245034) and The First Batch of High-level Talent Scientific Research Projects of the Affiliated Hospital of Youjiang Medical University for Nationalities in 2019 (grant no. R20196310).

\section{REFERENCES}

1. Jansen M, Yip S, Louis DN. Molecular pathology in adult gliomas: diagnostic, prognostic, and predictive markers. Lancet Neurol. 2010; 9:717-26. https://doi.org/10.1016/S1474-4422(10)70105-8 PMID:20610347

2. Reulen HJ, Poepperl G, Goetz C, Gildehaus FJ, Schmidt M, Tatsch K, Pietsch T, Kraus T, Rachinger W. Long-term outcome of patients with WHO Grade III and IV gliomas treated by fractionated intracavitary radioimmunotherapy. J Neurosurg. 2015; 123:760-70.

https://doi.org/10.3171/2014.12.JNS142168 PMID:26140493

3. Song $Y$, Luo $Q$, Long $H$, Hu Z, Que $T$, Zhang $X$, Li Z, Wang G, Yi L, Liu Z, Fang W, Qi S. Alpha-enolase as a potential cancer prognostic marker promotes cell growth, migration, and invasion in glioma. Mol Cancer. 2014; 13:65. 
https://doi.org/10.1186/1476-4598-13-65 PMID:24650096

4. Chen Z, Gao W, Pu L, Zhang L, Han G, Zuo X, Zhang Y, Li $\mathrm{X}$, Shen $\mathrm{H}$, Wu J, Wang X. PRDM8 exhibits antitumor activities toward hepatocellular carcinoma by targeting NAP1L1. Hepatology. 2018; 68:994-1009.

https://doi.org/10.1002/hep.29890 PMID:29572888

5. Queiroz CJS, Song F, Reed KR, Al-Khafaji N, Clarke AR, Vimalachandran D, Miyajima F, Pritchard DM, Jenkins JR. NAP1L1: A Novel Human Colorectal Cancer Biomarker Derived From Animal Models of Apc Inactivation. Front Oncol. 2020; 10:1565.

https://doi.org/10.3389/fonc.2020.01565 PMID: 32850460

6. Nagashio R, Kuchitsu $Y$, Igawa $S$, Kusuhara S, Naoki K, Satoh Y, Ichinoe M, Murakumo Y, Saegusa M, Sato Y. Prognostic significance of NAP1L1 expression in patients with early lung adenocarcinoma. Biomed Res. 2020; 41:149-59.

https://doi.org/10.2220/biomedres.41.149 PMID:32522932

7. Qiao H, Li Y, Feng C, Duo S, Ji F, Jiao J. Nap1l1 Controls Embryonic Neural Progenitor Cell Proliferation and Differentiation in the Developing Brain. Cell Rep. 2018; 22:2279-93. https://doi.org/10.1016/j.celrep.2018.02.019 PMID:29490266

8. Zhai W, Ma J, Zhu R, Xu C, Zhang J, Chen Y, Chen Z, Gong D, Zheng J, Chen C, Li S, Li B, Huang Y, et al. MiR-532-5p suppresses renal cancer cell proliferation by disrupting the ETS1-mediated positive feedback loop with the KRAS-NAP1L1/P-ERK axis. Br J Cancer. 2018; 119:591-604. https://doi.org/10.1038/s41416-018-0196-5 PMID: 30082686

9. Fujii-Nakata T, Ishimi $Y$, Okuda A, Kikuchi A. Functional analysis of nucleosome assembly protein, NAP-1. The negatively charged $\mathrm{COOH}$-terminal region is not necessary for the intrinsic assembly activity. J Biol Chem. 1992; 267:20980-6.

PMID: 1400414

10. Bagchi A, Orr BA, Campagne O, Dhanda S, Nair S, Tran Q, Christensen AM, Gajjar A, Furtado LV, Vasilyeva A, Boop F, Stewart C, Robinson GW. Lorlatinib in a Child with ALK-Fusion-Positive High-Grade Glioma. N Engl J Med. 2021; 385:761-3.

https://doi.org/10.1056/NEJMc2101264 PMID: 34407349

11. McKinnon C, Nandhabalan M, Murray SA, Plaha P. Glioblastoma: clinical presentation, diagnosis, and management. BMJ. 2021; 374:n1560. https://doi.org/10.1136/bmj.n1560

PMID:34261630

12. Chen J, Li Y, Yu TS, McKay RM, Burns DK, Kernie SG, Parada LF. A restricted cell population propagates glioblastoma growth after chemotherapy. Nature. 2012; 488:522-6. https://doi.org/doi: 10.1038/nature11287 PMID:22854781

13. Ohkuni K, Shirahige K, Kikuchi A. Genome-wide expression analysis of NAP1 in Saccharomyces cerevisiae. Biochem Biophys Res Commun. 2003; 306:5-9.

https://doi.org/10.1016/s0006-291x(03)00907-0 PMID: 12788058

14. Lee JY, Lake RJ, Kirk J, Bohr VA, Fan HY, Hohng S. NAP1L1 accelerates activation and decreases pausing to enhance nucleosome remodeling by CSB. Nucleic Acids Res. 2017; 45:4696-707. https://doi.org/10.1093/nar/gkx188 PMID:28369616

15. Simon HU, Mills GB, Kozlowski M, Hogg D, Branch D, Ishimi $Y$, Siminovitch KA. Molecular characterization of hNRP, a cDNA encoding a human nucleosomeassembly-protein-I-related gene product involved in the induction of cell proliferation. Biochem J. 1994; 297:389-97.

https://doi.org/10.1042/bj2970389

PMID:8297347

16. Kidd M, Modlin IM, Mane SM, Camp RL, Eick G, Latich I. The role of genetic markers--NAP1L1, MAGE-D2, and MTA1--in defining small-intestinal carcinoid neoplasia. Ann Surg Oncol. 2006; 13:253-62. https://doi.org/10.1245/ASO.2006.12.011 PMID:16424981

17. Line A, Slucka Z, Stengrevics A, Silina K, Li G, Rees RC. Characterisation of tumour-associated antigens in colon cancer. Cancer Immunol Immunother. 2002; 51:574-82. https://doi.org/10.1007/s00262-002-0322-2 PMID:12384809

18. Modlin IM, Kidd M, Latich I, Zikusoka MN, Eick GN, Mane SM, Camp RL. Genetic differentiation of appendiceal tumor malignancy: a guide for the perplexed. Ann Surg. 2006; 244:52-60.

https://doi.org/10.1097/01.sla.0000217617.06782.d5 PMID:16794389

19. Guidi F, Puglia M, Gabbiani C, Landini I, Gamberi T, Fregona D, Cinellu MA, Nobili S, Mini E, Bini L, Modesti PA, Modesti A, Messori L. 2D-DIGE analysis of ovarian cancer cell responses to cytotoxic gold compounds. Mol Biosyst. 2012; 8:985-93. https://doi.org/10.1039/c1mb05386h PMID:22134777 
20. Yang GY, Zhang $A Q$, Wang J, Li CH, Wang $X Q$, Pan $K$, Zhou C, Dong JH. Hepatoma-derived growth factor promotes growth and metastasis of hepatocellular carcinoma cells. Cell Biochem Funct. 2016; 34:274-85. https://doi.org/10.1002/cbf.3189

PMID:27273265

21. Yamamoto $S$, Tomita $Y$, Hoshida $Y$, Takiguchi $S$, Fujiwara Y, Yasuda T, Doki Y, Yoshida K, Aozasa K, Nakamura $\mathrm{H}$, Monden $\mathrm{M}$. Expression of hepatomaderived growth factor is correlated with lymph node metastasis and prognosis of gastric carcinoma. Clin Cancer Res. 2006; 12:117-22.

https://doi.org/10.1158/1078-0432.CCR-05-1347 PMID:16397032

22. Sun B, Gu X, Chen Z, Xiang J. MiR-610 inhibits cell proliferation and invasion in colorectal cancer by repressing hepatoma-derived growth factor. Am J Cancer Res. 2015; 5:3635-44.

PMID:26885452

23. Ren $H$, Tang $X$, Lee JJ, Feng L, Everett AD, Hong WK, Khuri FR, Mao L. Expression of hepatoma-derived growth factor is a strong prognostic predictor for patients with early-stage non-small-cell lung cancer. J Clin Oncol. 2004; 22:3230-7. https://doi.org/10.1200/JCO.2004.02.080 PMID:15310766

24. Song $Y, H u Z$, Long $H$, Peng $Y$, Zhang $X$, Que $T$, Zheng $S$, Li Z, Wang G, Yi L, Liu Z, Fang W, Qi S. A complex mechanism for HDGF-mediated cell growth, migration, invasion, and $\mathrm{TMZ}$ chemosensitivity in glioma. J Neurooncol. 2014; 119:285-95. https://doi.org/10.1007/s11060-014-1512-4 PMID:24986090

25. Lin X, Li AM, Li YH, Luo RC, Zou YJ, Liu YY, Liu C, Xie YY, Zuo S, Liu Z, Liu Z, Fang WY. Silencing MYH9 blocks $\mathrm{HBx}$-induced GSK3 $\beta$ ubiquitination and degradation to inhibit tumor stemness in hepatocellular carcinoma. Signal Transduct Target Ther. 2020; 5:13. https://doi.org/10.1038/s41392-020-0111-4 PMID:32296025

26. Min L, Ji Y, Bakiri L, Qiu Z, Cen J, Chen X, Chen L, Scheuch H, Zheng H, Qin L, Zatloukal K, Hui L, Wagner EF. Liver cancer initiation is controlled by AP-1 through SIRT6-dependent inhibition of survivin. Nat Cell Biol. 2012; 14:1203-11.

https://doi.org/10.1038/ncb2590

PMID:23041974

27. Zhao M, Xu P, Liu Z, Zhen Y, Chen Y, Liu Y, Fu Q, Deng $X$, Liang Z, Li Y, Lin X, Fang W. Dual roles of miR-374a by modulated c-Jun respectively targets CCND1inducing PI3K/AKT signal and PTEN-suppressing $W n t / \beta$-catenin signaling in non-small-cell lung cancer. Cell Death Dis. 2018; 9:78. https://doi.org/10.1038/s41419-017-0103-7 PMID:29362431

28. Ouafik L, Berenguer-Daize C, Berthois Y. Adrenomedullin promotes cell cycle transit and upregulates cyclin D1 protein level in human glioblastoma cells through the activation of c-Jun/JNK/AP-1 signal transduction pathway. Cell Signal. 2009; 21:597-608.

https://doi.org/10.1016/j.cellsig.2009.01.001 PMID:19166930

29. Ai B, Kong X, Wang X, Zhang K, Yang X, Zhai J, Gao R, Qi Y, Wang J, Wang Z, Fang Y. LINC01355 suppresses breast cancer growth through FOXO3-mediated transcriptional repression of CCND1. Cell Death Dis. 2019; 10:502.

https://doi.org/10.1038/s41419-019-1741-8 PMID:31243265

30. Liu $Y$, Jiang $Q$, Liu X, Lin X, Tang Z, Liu C, Zhou J, Zhao M, Li X, Cheng Z, Li L, Xie Y, Liu Z, Fang W. Cinobufotalin powerfully reversed EBV-miR-BART22induced cisplatin resistance via stimulating MAP2K4 to antagonize non-muscle myosin heavy chain IIA/glycogen synthase $3 \beta / \beta$-catenin signaling pathway. EBioMedicine. 2019; 48:386-404. https://doi.org/10.1016/j.ebiom.2019.08.040 PMID:31594754

31. Liu L, Ning Y, Yi J, Yuan J, Fang W, Lin Z, Zeng Z. miR6089/MYH9/ $\beta$-catenin/c-Jun negative feedback loop inhibits ovarian cancer carcinogenesis and progression. Biomed Pharmacother. 2020; 125:109865. https://doi.org/10.1016/j.biopha.2020.109865 PMID:32058212

32. Fu $Q$, Song $X$, Liu $Z$, Deng $X$, Luo R, Ge C, Li R, Li Z, Zhao $M$, Chen $Y$, Lin $X$, Zhang $Q$, Fang $W$. miRomics and Proteomics Reveal a miR-296-3p/PRKCA/FAK/ Ras/c-Myc Feedback Loop Modulated by HDGF/DDX5/ $\beta$-catenin Complex in Lung Adenocarcinoma. Clin Cancer Res. 2017; 23:6336-50. https://doi.org/10.1158/1078-0432.CCR-16-2813 PMID:28751441

33. Schindelin J, Arganda-Carreras I, Frise E, Kaynig V, Longair M, Pietzsch T, Preibisch S, Rueden C, Saalfeld S, Schmid B, Tinevez JY, White DJ, Hartenstein V, et al. Fiji: an open-source platform for biological-image analysis. Nat Methods. 2012; 9:676-82.

https://doi.org/10.1038/nmeth.2019 PMID:22743772

34. Xiao YY, Lin L, Li YH, Jiang HP, Zhu LT, Deng YR, Lin D, Chen $W$, Zeng $C Y$, Wang $\amalg$, Chen SC, Jiang $Q P$, Liu $C H$, et al. ZEB1 promotes invasion and metastasis of endometrial cancer by interacting with HDGF and inducing its transcription. Am J Cancer Res. 2019; 9:2314-30. PMID:31815037 
35. Zhao M, Luo R, Liu Y, Gao L, Fu Z, Fu Q, Luo X, Chen $Y$, Deng $X$, Liang Z, Li X, Cheng C, Liu Z, Fang W. miR-3188 regulates nasopharyngeal carcinoma proliferation and chemosensitivity through a FOXO1-modulated positive feedback loop with mTOR-p-PI3K/AKT-c-JUN. Nat Commun. 2016; 7:11309.

https://doi.org/10.1038/ncomms11309

PMID:27095304 


\section{SUPPLEMENTARY MATERIALS}

\section{Supplementary Figures}

A

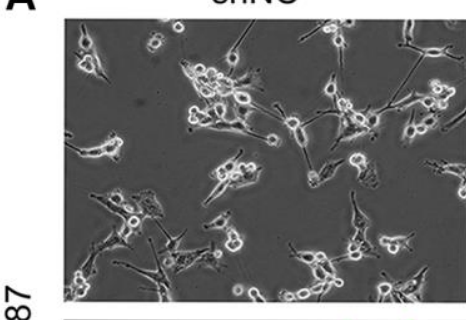

$\stackrel{Ð}{\supset}$
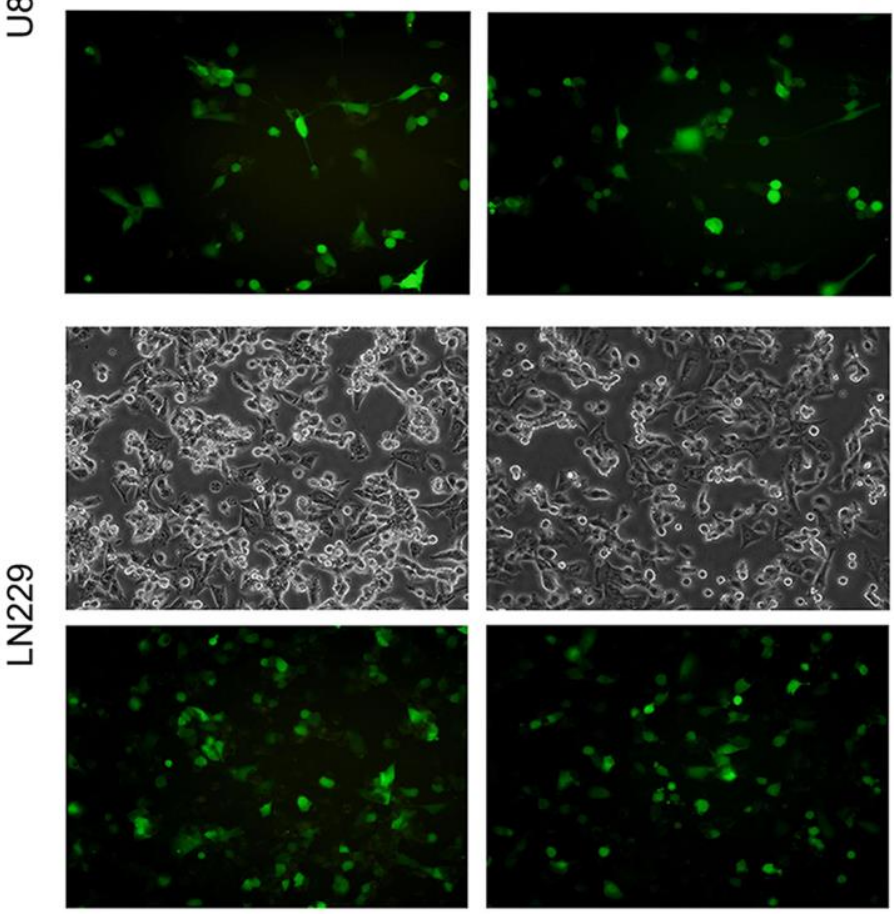

shNAP\#1
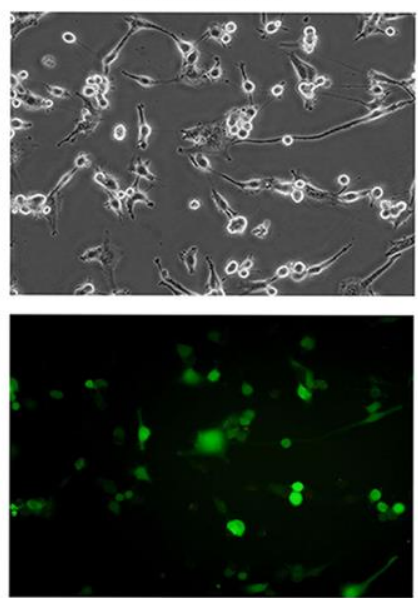
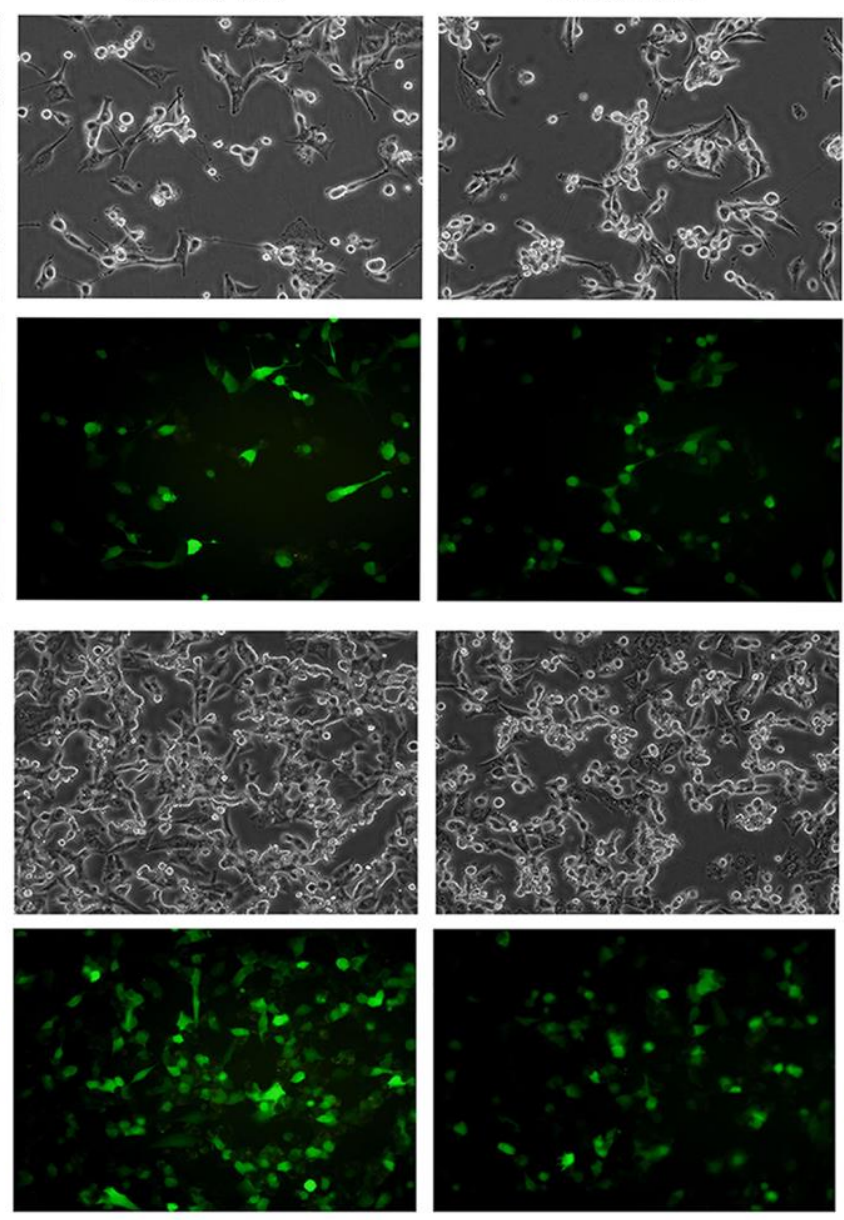

B

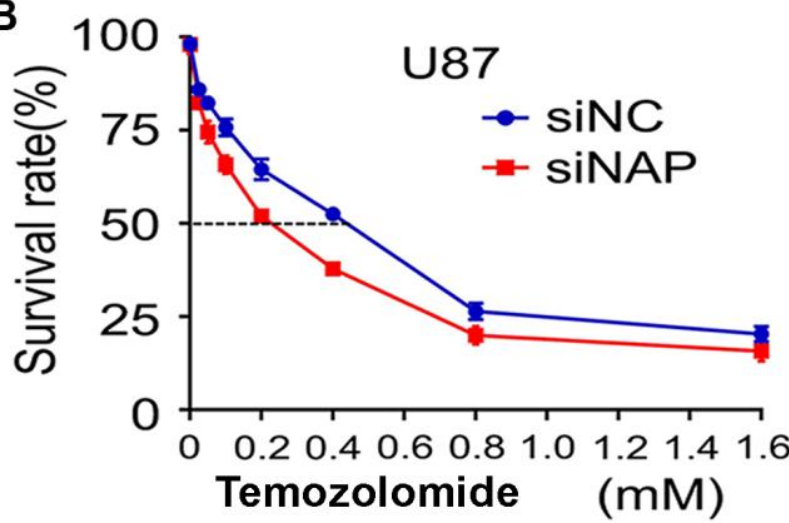

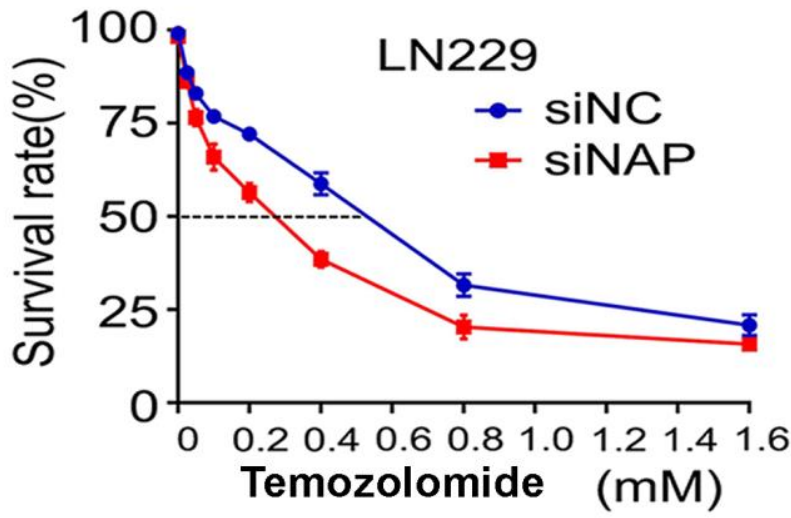

Supplementary Figure 1. (A) Representative images of U87 and LN229 cells after transfecting lentiviruses containing shNC or shNAP. Scale bar: $25 \mu \mathrm{m}$. (B) Dose-response curves of U87 and LN229 treated with shNAP and shNC respectively following treatment with temozolomide for $48 \mathrm{~h}$. Data are presented as the mean \pm SD for three independent experiments. ${ }^{*} P<0.05,{ }^{* *} P<0.01,{ }^{* * *} P<0.001$. 
A U87

LN229
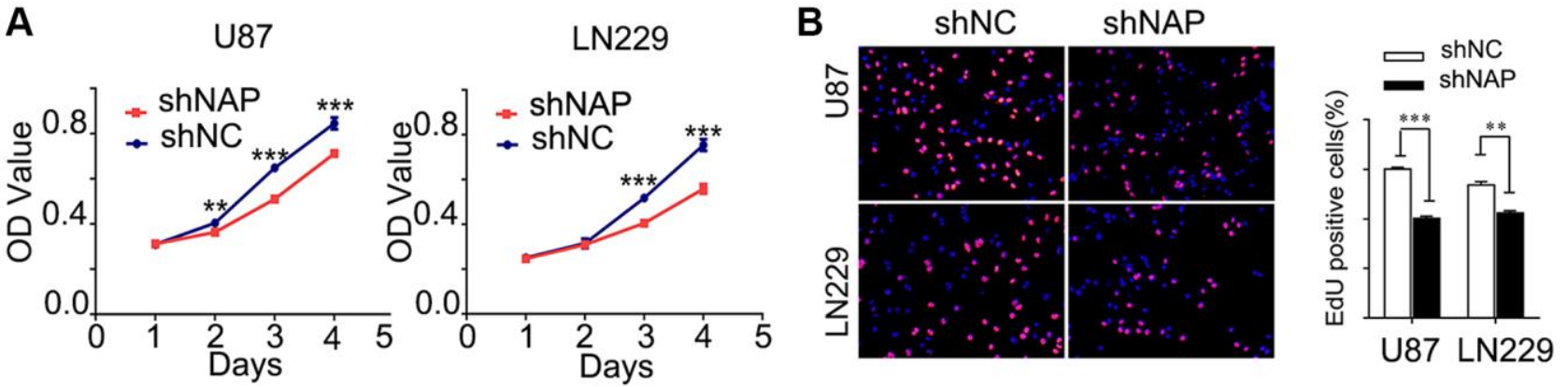

C
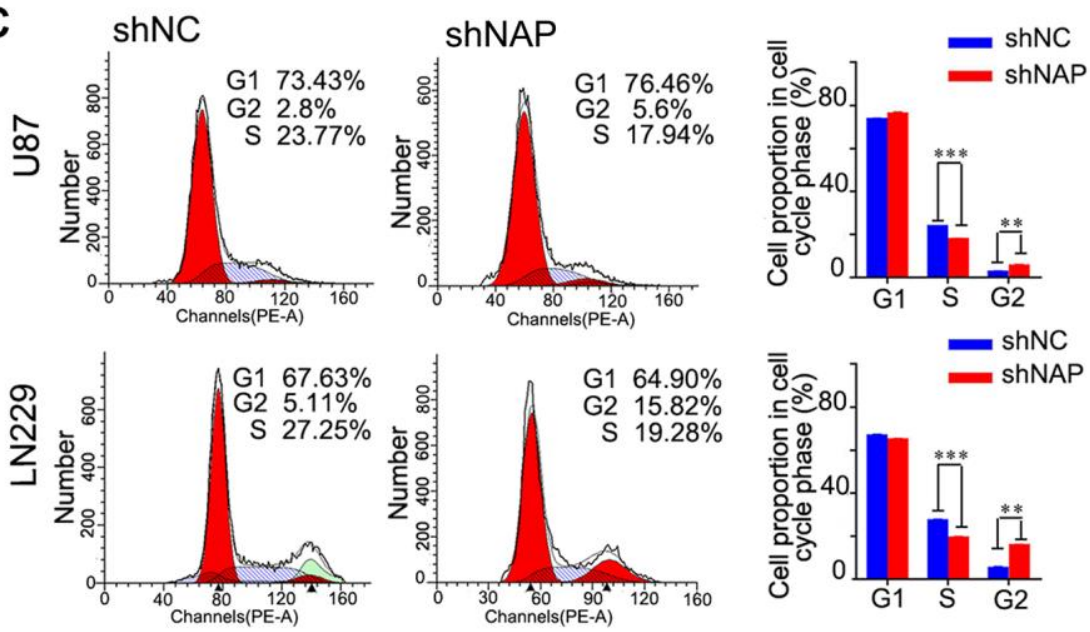

D

U87

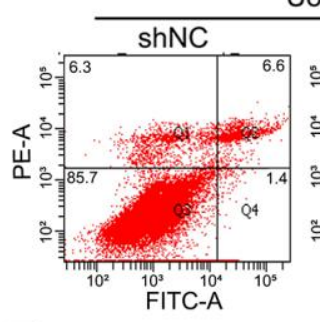

E

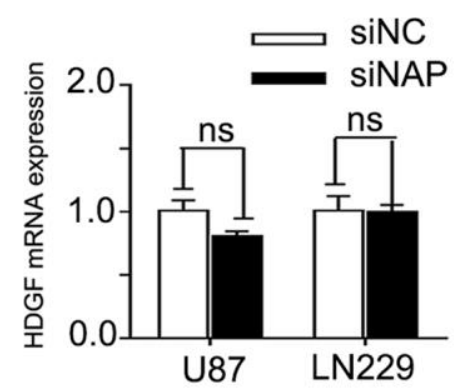

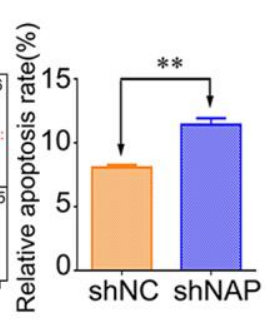

F

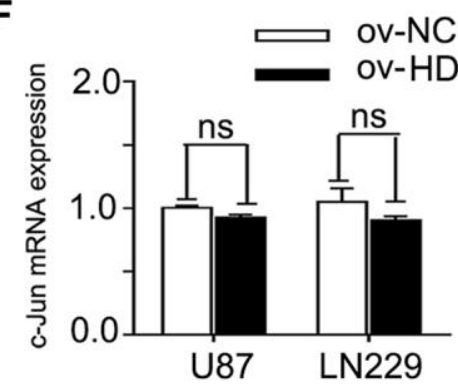

LN229

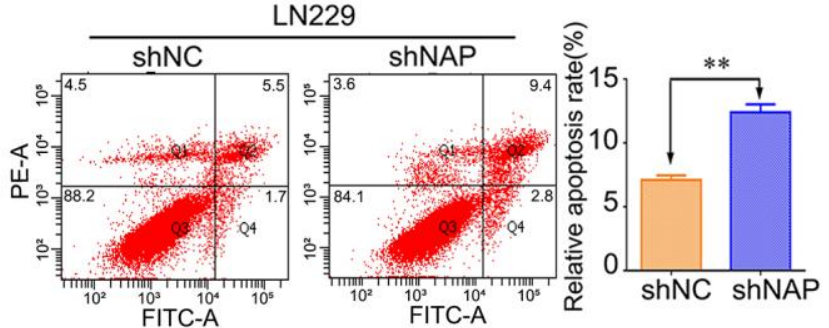

G

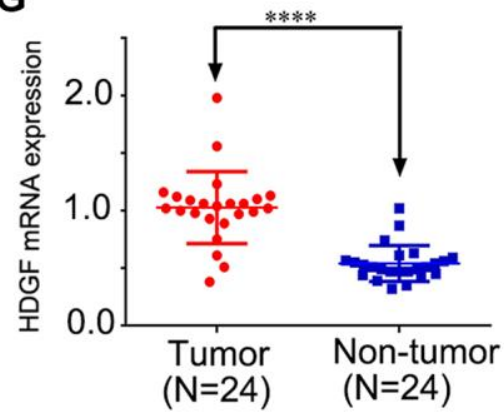

Supplementary Figure 2. Suppressing NAP1L1 inhibits glioma cell proliferation, blocks the cell cycle and induces apoptosis. (A) U87 and LN229 cells were transfected with shNC or shNAP lentiviruses, and then subjected to MTT assay. (B) The proliferation of U87 and LN229 cells transfected with lentiviruses containing shNC or shNAP is measured using the EdU assays. (C, D) The cell cycle distribution (C) and apoptotic fraction (D) in U87 and LN229 cells transfected with lentiviruses containing shNC or shNAP is analyzed by flow cytometry. (E) RT-qPCR analysis of HDGF mRNA expression in U87 and LN229 cells transfected with siNAP1L1. (F) RT-qPCR analysis of c-Jun mRNA level in U87 and LN229 cells transfected with HDGF-overexpressing plasmid. (G) RT-qPCR analysis of HDGF mRNA expression in 24 glioma tissues and 24 para-tumor tissues. Data are presented as the mean \pm SD for three independent experiments. ${ }^{*} P<0.05,{ }^{* *} P<0.01,{ }^{* * *} P<0.001$. 


\section{Supplementary Tables}

Supplementary Table 1. shRNA sequences of NAP1L1.

\begin{tabular}{|c|c|c|c|c|c|}
\hline NO. & $5^{\prime}$ & STEM & Loop & STEM & $\mathbf{3}^{\prime}$ \\
\hline NAP1L1-RNAi(78814)-a & Ccgg & $\begin{array}{c}\text { gcCAAGATTGAAGA } \\
\text { TGAGAAA }\end{array}$ & CTCGAG & $\begin{array}{c}\text { TTTCTCATCTTCA } \\
\text { ATCTTGGC }\end{array}$ & TTTTTg \\
\hline NAP1L1-RNAi(78814)-b & aattcaaaaa & $\begin{array}{c}\text { gcCAAGATTGAAGA } \\
\text { TGAGAAA }\end{array}$ & CTCGAG & $\begin{array}{c}\text { TTTCTCATCTTCA } \\
\text { ATCTTGGC }\end{array}$ & \\
\hline NAP1L1-RNAi(78815)-a & Ccgg & $\begin{array}{l}\text { TTGATAAGCGATTT } \\
\text { GAAATTA }\end{array}$ & CTCGAG & $\begin{array}{l}\text { TAATTTCAAATCG } \\
\text { CTTATCAA }\end{array}$ & TTTTTg \\
\hline NAP1L1-RNAi(78815)-b & aattcaaaaa & $\begin{array}{l}\text { TTGATAAGCGATTT } \\
\text { GAAATTA }\end{array}$ & CTCGAG & $\begin{array}{l}\text { TAATTTCAAATCG } \\
\text { CTTATCAA }\end{array}$ & \\
\hline NAP1L1-RNAi(78816)-a & Ccgg & $\begin{array}{c}\text { TTCCAATGACTCTT } \\
\text { TCTTTAA }\end{array}$ & CTCGAG & $\begin{array}{l}\text { TTAAAGAAAGAG } \\
\text { TCATTGGAA }\end{array}$ & TTTTTg \\
\hline NAP1L1-RNAi(78816)-b & aattcaaaaa & $\begin{array}{c}\text { TTCCAATGACTCTT } \\
\text { TCTTTAA }\end{array}$ & CTCGAG & $\begin{array}{l}\text { TTAAAGAAAGAG } \\
\text { TCATTGGAA }\end{array}$ & \\
\hline
\end{tabular}

Supplementary Table 2. siRNA sequences of NAP1L1 and HDGF.

\begin{tabular}{lll}
\hline Gene & NO & Target Sequence \\
\hline NAP1L1 & genOFFTM st-h-NAP1L1_001 & GAAGTATGCTGTTCTCTAT \\
& genOFFTM st-h-NAP1L1_002 & CCAACAGGATACATTGAAA \\
genOFFTM st-h-NAP1L1_003 & GACAGTTCGTACTGTGACT \\
HDGF & HDGF-RNAi-1 & 5'GAAACGAGAUCGAAUGCAC dTdT 3' \\
& HDGF-RNAi-2 & 5'CUCAAGCGUUUCCUCCUUA dTdT 3' $^{\prime}$ \\
& HDGF-RNAi-3 & 5'CCAUACGAUUGACGAGAUG dTdT 3' $^{\prime}$ \\
\hline
\end{tabular}

Supplementary Table 3. The primers used in this study.

\begin{tabular}{lll}
\hline Primers name & & Sequence $\left(\mathbf{5}^{\prime} \mathbf{-} \mathbf{3}^{\prime}\right)$ \\
\hline NAP1L1 & Forward & TTTGCCCCTCCTGAAGTTCC \\
& Reverse & CCCAACACAACTTGAGACATCC \\
HDGF & Forward & ATCAACAGCCAACAAATACC \\
& Reverse & TTCTTATCACCGTCACCCT \\
c-JUN & Forward & TCAGACAGTGCCCGAGATG \\
& Reverse & CTGCTGCGTTAGCATGAGTT \\
GAPDH & Forward & CATGGGTGTGAACCATGAGA \\
& Reverse & GTCTTCTGGGTGGCAGTGAT \\
\hline
\end{tabular}


Supplementary Table 4. A list of antibodies used for WB, IF, CoIP and IHC.

\begin{tabular}{lllll}
\hline Antibody & Cat. No & Company & Species & Dilution \\
\hline NAP1L1 & mAb ab 178687 & Abcam & Rabbit & $1: 1000(\mathrm{WB}) ; 1: 200(\mathrm{IF}) ; 1: 20(\mathrm{CoIP})$ \\
NAP1L1 & ab 33076 & Abcam & Rabbit & $1: 1000(\mathrm{WB}) ; 1: 100(\mathrm{IF}) ; 1: 300(\mathrm{IHC})$ \\
NAP1L1 & pAb \#14898-1-AP & Proteintech & Rabbit & $1: 1000(\mathrm{WB}) ; 1: 100(\mathrm{IF}) ; 1: 300(\mathrm{IHC})$ \\
HDGF & mAb \#60064-1-Ig & Proteintech & Mouse & $1: 1000(\mathrm{WB}) ; 1: 50(\mathrm{IF}) ; 1: 10(\mathrm{CoIP})$ \\
HDGF & pAb 11344-1-AP & Proteintech & Rabbit & $1: 1000(\mathrm{WB}) ; 1: 200(\mathrm{IHC})$ \\
c-Jun & mAb \#9165 & Cell Sigaling & Rabbit & $1: 1000(\mathrm{WB}) ; 1: 50(\mathrm{IF}) ; 1: 10(\mathrm{CoIP})$ \\
c-Jun & 24909-1-AP & Proteintech & Rabbit & $1: 1000(\mathrm{WB}) ; 1: 100(\mathrm{IF}) ; 1: 10(\mathrm{CoIP})$ \\
c-Jun & 28891-1-AP & Proteintech & Mouse & $1: 1000(\mathrm{WB}) ; 1: 100(\mathrm{IF}) ; 1: 10(\mathrm{CoIP})$ \\
CCND1 & mAb \#6086-1-Ig & Proteintech & Mouse & $1: 1000(\mathrm{WB})$ \\
PCNA & mAb \#13110 & Cell Sigaling & Rabbit & $1: 300(\mathrm{IHC})$ \\
GAPDH & pAb AP0063 & Bioworld & Rabbit & $1: 1000(\mathrm{WB})$ \\
BCL2 & ab182858 & Abcam & Rabbit & $1: 1000(\mathrm{WB})$ \\
Ki-67 & Ab16667 & Abcam & Mouse & $1: 200(\mathrm{IHC})$ \\
CDK4 & ab199728 & Abcam & Rabbit & $1: 1000(\mathrm{WB})$ \\
CDK6 & ab241554 & Abcam & Mouse & $1: 1000(\mathrm{WB})$ \\
Cleaved & Ab214430 & Abcam & Rabbit & $1: 1000(\mathrm{WB})$ \\
Caspase3 & & & & \\
\hline
\end{tabular}

